\title{
Caracterización petrofísica como potencial reservorio de las sucesiones siliciclásticas paleógenas del pozo ANH- TIERRALTA-2-X-P en la Cuenca Sinú-San Jacinto, caribe colombiano
}

\author{
Cristian Camilo Manrique-Gómez ${ }^{*}$, Carlos Alberto Guzmán-López ${ }^{1}$, Robinson Aguirre²
}

doi: http://dx.doi.org/10.18273/revbol.v42n1-2020001 (c) (1)

Forma de citar: Manrique-Gómez, C.C., Guzmán-López, C.A., y Aguirre, R. (2020). Caracterización petrofísica como potencial reservorio de las sucesiones siliciclásticas paleógenas del pozo ANH-TIERRALTA-2-X-P en la Cuenca Sinú-San Jacinto, caribe colombiano. Boletín de Geología, 42(1), 15-37. doi: 10.18273/revbol.v42n12020001.

\section{RESUMEN}

Una evaluación petrofísica se llevó a cabo para los niveles siliciclásticos del pozo ANH-TIERRALTA-2-X-P en la Cuenca de Sinú-San Jacinto, Noroeste de Colombia. El pozo se dividió en cinco unidades informales que contienen diez ciclos estratigráficos basándose en la litología y la respuesta del registro de gamma ray. La primera desde la superficie hasta $1100 \mathrm{ft}$ (Formación El Carmen, Oligoceno), la segunda (1100ft - $3100 \mathrm{ft}$ ) compuesta de biomicritas (Formación Tolúviejo, Eoceno). La Unidad 3 corresponde a areniscas cuarzosas y litoarenitas interestratificadas con lutitas (de $2000 \mathrm{ft}$ de espesor); la Unidad 4 corresponde a areniscas conglomeráticas $(5530 \mathrm{ft}-6620 \mathrm{ft})$; la Unidad $5(6620 \mathrm{ft}$ hasta $6770 \mathrm{ft})$ contiene calizas grises; estas tres últimas, se correlacionan con la Formación San Cayetano, Paleoceno. La unidad basal (6770 ft hasta $8711 \mathrm{ft}$ ) contiene lutitas de color gris oscuro, y se correlaciona con la Formación Cansona, del Cretácico superior. Petrográficamente, se analizaron 26 secciones delgadas del Ciclo 8 (Unidad 3). La diagénesis temprana está representada por compactación, caolinitización de feldespatos alcalinos y sericitización de plagioclasas, y la diagénesis de enterramiento por neoformación de cemento de poros de calcita. Los ciclos siliciclásticos basales (4, 5 y 6 en la Unidad 4) son los más prospectivos para la acumulación de hidrocarburos, mientras que el Ciclo 7 (en la Unidad 3), correspondería a la roca sello del posible sistema petrolífero. El volumen de shale se calculó a partir de los registros de gamma ray y densidad-neutrón. Los cálculos de porosidad hechos a partir del registro de densidad (entre 8 y $15 \%$ ) son los más próximos a los valores calculados en el laboratorio a partir de los plugs recuperados (5\%). La saturación de agua se estimó utilizando la porosidad calculada y el registro de resistividad profunda, reportándose un valor promedio de $95 \%$ en los potenciales reservorios analizados.

Palabras clave: Sinú-San Jacinto; petrografía; diagénesis; petrofísica.

\section{Petrophysical characterization as a potential reservoir of the siliciclastic paleogenes sucesions of the ANH-TIERRALTA-2-X-P well in the Sinú-San Jacinto Basin, colombian Caribbean}

\begin{abstract}
A petrophysical evaluation was carried out for the siliciclastic levels of the ANH-TIERRALTA-2-X-P well in the Sinú-San Jacinto Basin, Northwest of Colombia. The well was divided into five informal units that contain ten stratigraphic cycles based on the lithology and response of the gamma ray log. The first from surface to $1100 \mathrm{ft}$ (El Carmen Formation, Oligocene), the second (1100 ft - $3100 \mathrm{ft}$ ) composed of biomicrites (Tolúviejo Formation, Eocene). Unit 3 corresponds to quartzitic and lithic sandstones interbedded with shales (2000 ft thick); Unit 4 corresponds to conglomerate sandstones (5530 ft - $6620 \mathrm{ft}$ ); Unit 5 $(6620 \mathrm{ft}$ to $6770 \mathrm{ft}$ ) contains gray limestones; these last three, correlate with the San Cayetano Formation, from Paleocene. The basal unit (6770 ft to $8711 \mathrm{ft}$ ) contains dark gray shales, and correlates with the Cansona Formation of the Upper Cretaceous. Petrographically, 26 thin sections of Cycle 8 (Unit 3) were analyzed. Early diagenesis is represented by compaction, alkali feldspar kaolinitization and plagioclase sericitization, and the burial diagenesis by neoformation of calcite cement. The basal siliciclastic cycles (4, 5 and 6 in Unit 4) are the most prospective for the accumulation of hydrocarbons, while Cycle 7 (in Unit 3) will correspond to the cap rock of the possible oil system. The shale volume was calculated from the gamma ray and density-neutron logs. The porosity calculations made from the density $\log$ (between 8 and 15\%) are the closest to the values calculated in the laboratory from the recovered plugs $(5 \%)$. The water saturation was estimated using the calculated porosity and the deep resistivity log, reporting an average value of $95 \%$ in potential reservoirs analyzed.
\end{abstract}

Keywords: Sinú-San Jacinto; petrography; diagenesis; petrophysics.

${ }^{1}$ Instituto de Investigaciones en Estratigrafía, Universidad de Caldas, Manizales, Colombia.

(*) cristian.manrique.gomez@hotmail.com; carlosguzman@ucaldas.edu.co

${ }^{2}$ ECOPETROL, Bogotá, Colombia. robinson.aguirre@ecopetrol.com.co 


\section{INTRODUCCIÓN}

En la parte noroccidental de Colombia se tiene la Cuenca de Sinú-San Jacinto (Figura 1) que presenta una de las mayores manifestaciones de hidrocarburos en superficie del país (ANH, 2016), en virtud de esta situación se hace importante entender y estudiar cómo es el sistema petrolífero asociado, con el fin de efectuar una exploración con más elementos de juicio.

Los pozos Tubará-1 y Sinú-1 perforados en los años 80 's permitieron establecer la presencia de rocas generadoras, asociadas con lutitas de la Formación Cansona (Cretácico superior) con un contenido de materia orgánica que varía entre 2 y $11 \%$ y kerógenos tipo I y II principalmente (Beroiz et al., 1986). El pozo Caracoli-1 atravesó la Formación San Cayetano (Paleoceno) y a partir de información geoquímica se reportaron rocas con valores de TOC entre 0,4 y $1 \%$ y kerógenos tipo III y IV (Beroiz et al., 1986).

Posteriormente, en el año 2014, el pozo ANHTIERRALTA-2-X-P, perforó la principal estructura de la cuenca, el denominado Anticlinorio de San Jerónimo (Figura 1). La información obtenida reveló la existencia de más de $3000 \mathrm{ft}$ de biomicritas y shales del Cretácico superior - Paleoceno con un TOC superior a 5\% y kerógenos tipo II característicos de ambientes de plataforma externa en medios anóxicos (ANH, 2016); así mismo, se reportaron ciclos capaces de albergar hidrocarburos.

Si bien se dispone de alguna información geoquímica, bioestratigráfica y sedimentológica, la información petrofísica es muy poca, por lo que se busca realizar un estudio a partir de catorce plugs tomados de núcleos recuperados y de registros eléctricos obtenidos del pozo ANH-TIERRALTA-2-X-P. El propósito fundamental es evaluar las propiedades petrofísicas de las sucesiones siliciclásticas y el efecto de la textura, composición y diagénesis sobre ellas.

\section{LOCALIZACIÓN Y GENERALIDADES}

El pozo ANH-TIERRALTA-2-X-P está ubicado en la parte SW de la Cuenca Sinú-San Jacinto en el Caribe colombiano (Figura 1) con las siguientes coordenadas origen Magna Sirgas Bogotá: Norte: 1395282,417 m, Este: $796997,053 \mathrm{~m}$.

Al pozo se accede tomando la vía Departamental que va de Medellín a Montería. Antes de llegar a la cabecera municipal de Tierralta, se toma la variante para Urrá, buscando a 3,3 km el desvío hacia la izquierda por la vía secundaria en buen estado Cerro Guillo - El Pando o Nueva Granada (ANH, 2014a). El pozo ANH-TIERRALTA-2-X-P, es un pozo estratigráfico exploratorio, perforado en tres fases, hasta una profundidad final de $8,711 \mathrm{ft}$ profundidad medida (MD), se corazonaron $1867 \mathrm{ft}$ de los cuales se recuperaron en superficie $1845,24 \mathrm{ft}$, para un porcentaje de recobro del $98,83 \%$; se tomaron muestras de zanja húmeda y seca cada $20 \mathrm{ft}$ hasta $6963 \mathrm{ft}$ y cada $10 \mathrm{ft}$ a partir de esta profundidad, además se adquirieron e interpretaron registros eléctricos en las tres fases perforadas.

\section{MARCO GEOLÓGICO}

La Cuenca Sinú-San Jacinto se caracteriza por estar sometida a tectónica transpresiva que genera una serie de fallas inversas con vergencia al oeste y rumbo dextral, sobre las que se desarrollan pliegues estrechos y alargados (Figura 1) que involucran una secuencia sedimentaria despositada desde finales del Cretácico (Bermúdez et al., 2009).

Las rocas sedimentarias más antiguas de la zona, lodolitas calizas y cherts de la Formación Cansona (CampanianoMaastrichtiano), se depositaron en forma discordante sobre un basamento de naturaleza continental a transicional (Figura 2); al tope de esta secuencia marina se presenta un proceso de somerización como lo mencionan trabajos anteriores hechos por la Agencia Nacional de Hidrocarburos (Universidad de Caldas-ANH, 2011), esta somerización lleva a una sedimentación clástica basal donde predominan ambientes litorales con secuencias de areniscas cuarzosas de tamaño medio a grueso con porosidades mayores al $15 \%$ muestreadas en pozos slimhole perforados por la ANH. Sobre estas secuencias basales, se depositó una espesa secuencia de arcillolitas y lodolitas calcáreas depositadas en ambientes transicionales que sirven como sello para el reservorio siliciclástico basal. Hacia el tope de estas secuencias se presenta un cambio gradual desde litoarenitas de grano fino a cuarzoarenitas de grano medio a grueso de ambientes deltaicos datados por bioestratigrafia en el Eoceno inferior que pertenecen a la Formación San Cayetano (ANH, 2016).

Para el Eoceno medio (Figura 2) se desarrollaron sistemas de calizas algáceas depositadas sobre estas secuencias deltaicas como la Formación Chenge y equivalentes (Formación Tolúviejo) (Guzmán, 2007). Esta depositación se presentó en todo el Caribe colombiano encontrándose espesores de hasta $2000 \mathrm{ft}$ de calizas arrecifales, biomicritas y calizas algáceas con alto contenido de foraminíferos tipo Nummulites (ANH, 2016). 

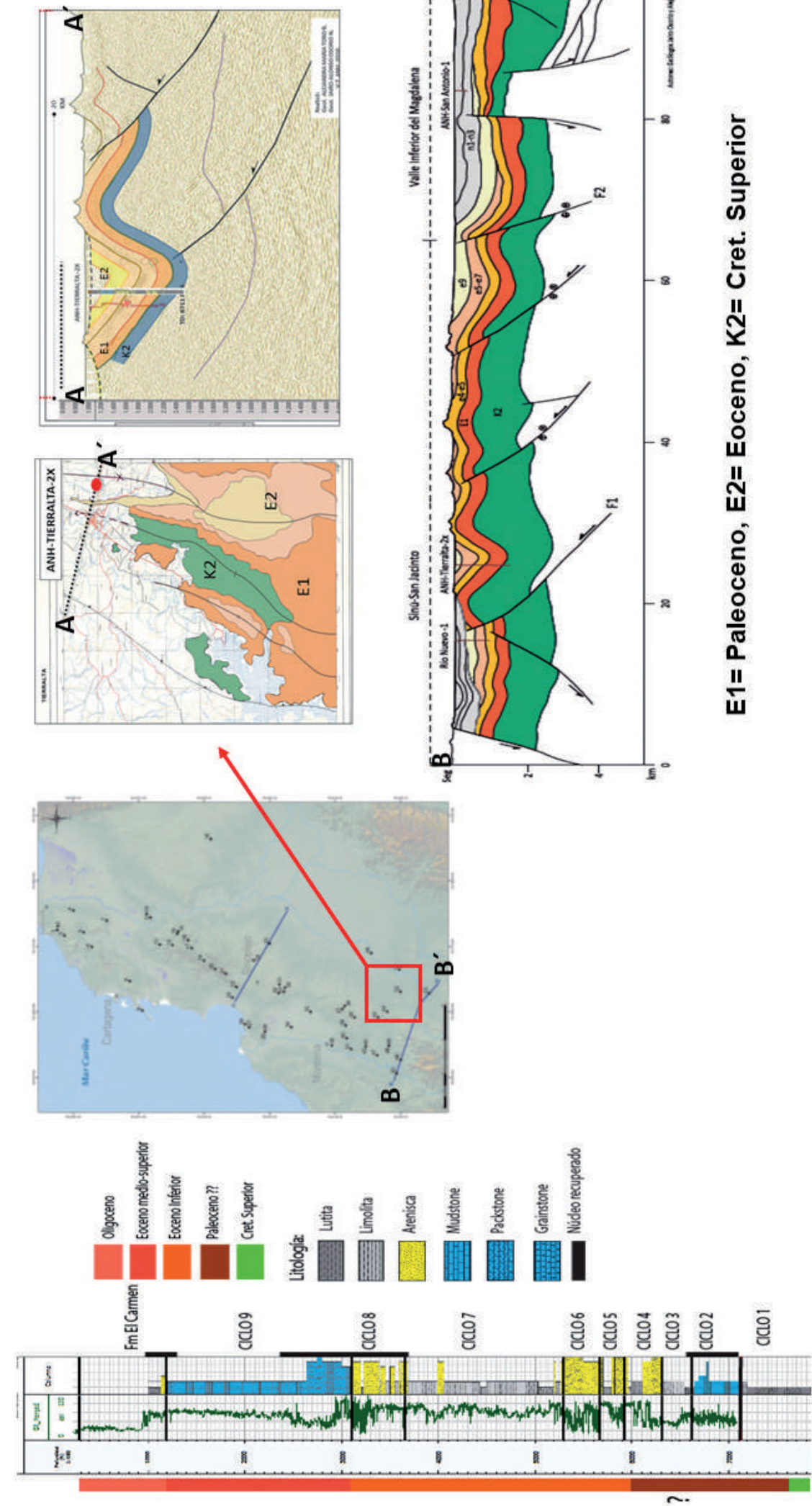

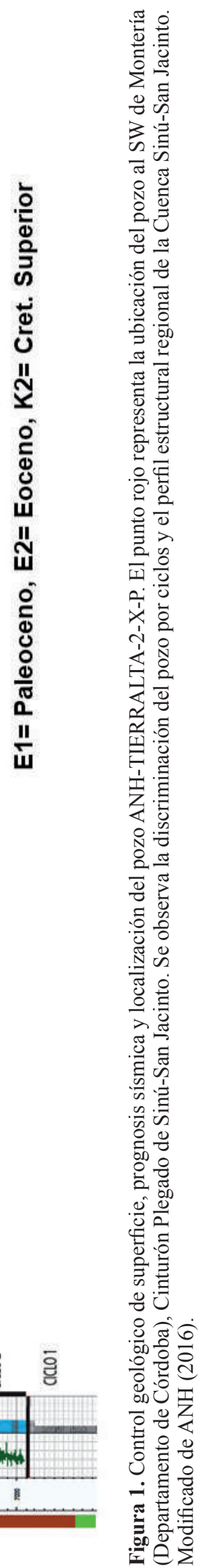




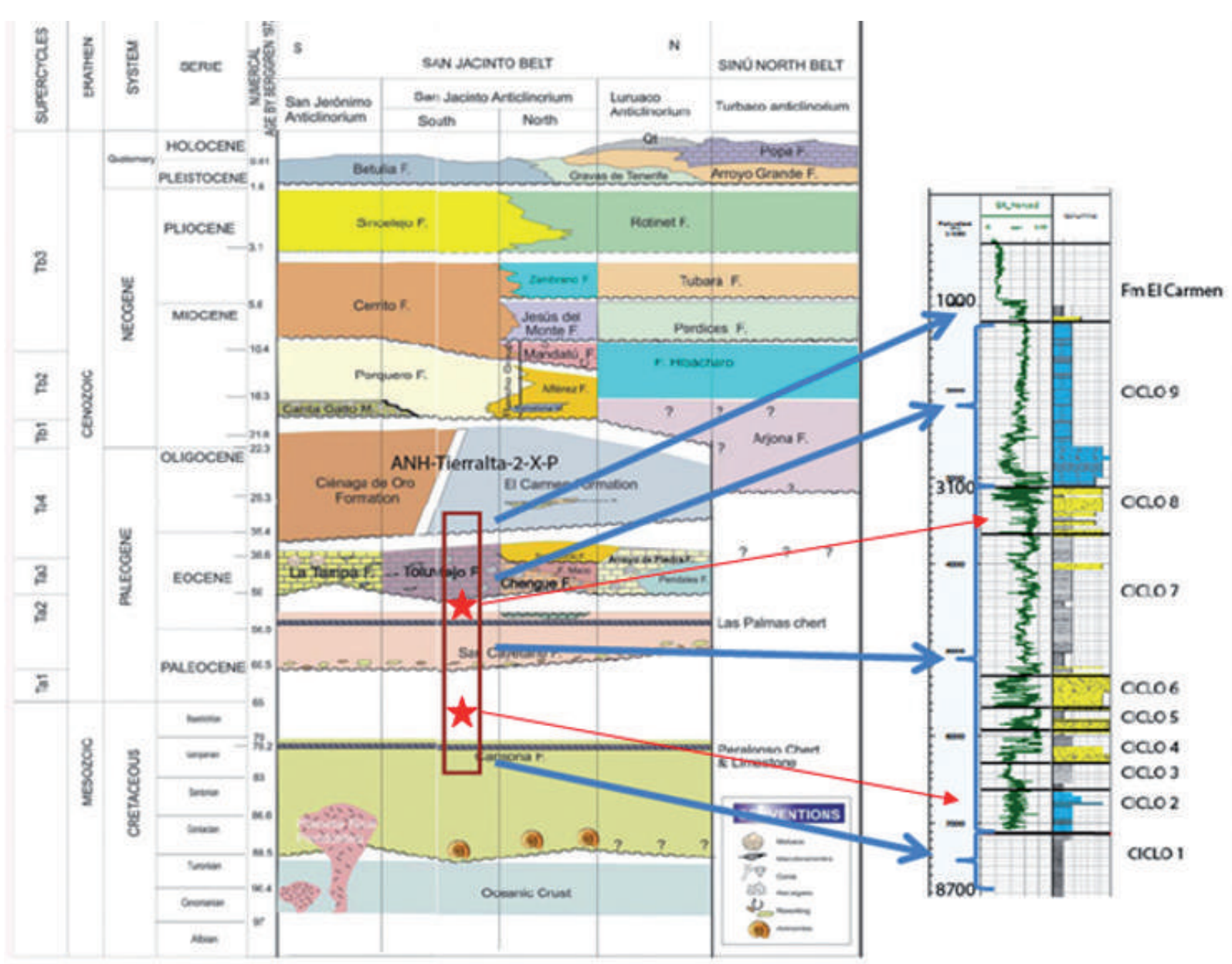

Figura 2. Cuadro cronoestratigráfico del Cinturón de San Jacinto y Cinturón del Sinú. Modificado de Guzmán (2007). Se muestra la ubicación estratigráfica del pozo ANH-TIERRALTA-2-X-P en la zona sur del Anticlinorio de San Jacinto, las estrellas rojas indican la posición estratigráfica de los ciclos analizados.

En el Oligoceno se depositaron secuencias finas las cuales corresponden a secuencias de crecimiento que se correlacionan con la Formación el Carmen (Guzmán, 2007).

El Plioceno es caracterizado por las facies continentales de la Formación Sincelejo. Esta unidad está compuesta principalmente por limolitas bioturbadas, alto contenido de moscovita y carbón (Alfaro y Holz, 2014).

\section{Estratigrafía del Cinturón de San Jacinto}

Está constituida por shales, margas, cherts y calizas micríticas de color gris oscuro a negro de ambientes batiales (Guzmán, 2007). Bermúdez et al. (2009) sugieren una edad de Campaniano-Maastrichtiano a partir de palinología, foraminíferos y nanopláncton calcáreo, en el sector de Chalán, Departamento de Sucre.

\section{Formación San Cayetano}

Nombrada por Chenevart (1963 en Guzmán, 2007) a una unidad compuesta por lutitas pobremente fosilíferas verde oliva grisáceas interestratificada con limolitas micáceas y en algunos niveles interestratificada con areniscas gruesas a conglomerados. Su espesor varía grandemente $(600$ a $1200 \mathrm{~m})$ y ha sido afectada por varios sistemas de fallas (Guzmán, 2007). Duque-Caro (1984 en Guzmán et al., 2004) indica que la fauna de la Formación San Cayetano corresponde a las zonas planctónicas P.3 a P.9 y a las zonas bentónicas de Rzehakina epigona y Spiroplectamina y sugiere una edad Paleoceno tardío - Eoceno temprano.

\section{Formación Maco}

Nombre dado por Butler (1942 en Guzmán, 2007) a una serie de conglomerados y areniscas expuestas en 
el Cerro Maco. En la parte inferior de la unidad se presenta una discordancia suprayacida por capas de chert. Es posible que las capas de chert representen el Eoceno inferior basándose en el contenido fósil (Guzmán, 2007). El espesor de la Formación Maco es aproximadamente de $900 \mathrm{~m}$ en el Cerro Maco (Guzmán, 2007).

\section{Formación Tolúviejo}

Fue definida por Werenferls (1926 en Villafrade, 2007). Duque-Caro (2000) le otorgó una edad de Eoceno medio a superior con base a la fauna encontrada correspondiente a las zonas planctónicas P.10 a P.18 y a la zona bentónica Bulimina jacksonensis. La Formación Tolúviejo yace en discordancia angular sobre la Formación San Cayetano (Figura 2). Consta de margas y limolitas calcáreas de colores oscuros que varían a areniscas grises de grano fino a medio, glauconíticas y con cemento calcáreo. Hacia el tope, tiene calizas de color crema, compactas y con abundantes restos fósiles de corales, equinodermos, algas, etc.

\section{Formación El Carmen}

La Formación El Carmen está presente en el área este de la serranía de San Jacinto, en el Valle inferior del Magdalena. Fue nombrada por Notestein y Aitken (1929 en Guzmán, 2007). Está compuesta por una arcillolita color gris oscuro; hacia la base de la formación hay presencia de areniscas arcillosas y lutitas arenosas (Guzmán, 2007). El espesor promedio para la Formación El Carmen es de $586 \mathrm{~m}$, y su fauna corresponde a las zonas planctónicas P.19 a la P.20/P.21 y a la zona bentónica de Cibicides perlucida, indicando una edad Oligoceno temprano-Mioceno temprano (Guzmán et al., 2004).

\section{METODOLOGÍA}

Se hizo un inventario de todos los registros del pozo ANH-TIERRALTA-2-X-P, esto con el fin de hacer un análisis básico de las propiedades petrofísicas de las formaciones perforadas. Se elaboraron 26 secciones delgadas para el Ciclo 8 (intervalo siliciclástico muestreado). Se analizaron los datos petrográficos con el objetivo de interpretar el contexto sedimentario de las unidades muestreadas a partir de los núcleos de roca. Para cada sección delgada se realizó un conteo de 300 puntos, es importante aclarar que solo se hizo la interpretación de los niveles siliciclásticos del pozo, los niveles carbonatados no fueron objeto de estudio en este trabajo. Para rocas terrígenas se tuvo en cuenta con respecto a la textura: tamaño de grano promedio, porcentaje y tipo de matriz, selección y redondez. Las muestras fueron analizadas y clasificadas siguiendo la propuesta de Folk (1974) para la clasificación de arenitas tanto textural como composicionalmente. Se hizo la identificación y establecimiento de los principales procesos, productos diagenéticos y la sucesión de eventos.

El trabajo específico sobre la diagénesis se hizo teniendo presente las transformaciones del espacio intersticial con respecto a la porosidad, poronecrosis y compactación, se establecieron neoformaciones minerales y metasomatismos diagenéticos. Se estudiaron transformaciones de los componentes (corrosión, disolución y neomorfismo) y finalmente se determinó la paragénesis diagenética a partir de lo observado en las secciones delgadas considerando las relaciones entre los productos diagenéticos con el fin de establecer una secuencia relativa de eventos.

Por último, se hizo la carga de datos en formato .las de los registros eléctricos del pozo ANH-TIERRALTA2-X-P en el software "Techlog" versión 2011.2 de la compañía Schlumberger. La información fue cargada y revisada con el objetivo de seleccionar únicamente las curvas originales (no sintéticas) del pozo: estas curvas se almacenaron en un conjunto de datos o "data sets" que correspondieron a los registros usados en los diferentes análisis hechos en este trabajo.

Se hizo una columna estratigráfica general (Figura 3) con base en el registro de perforación del pozo (registro gráfico compuesto) y se dividió en ciclos estratigráficos, basándose en su litología y respuesta del registro de gamma ray. Para todos los intervalos silisiclásticos se hizo un análisis sobre el contenido de arcilla, mineralogía, y saturación de fluidos. Se calculó el volumen de shale $\left(V_{s h}\right)$ utilizando el método lineal y usando los registros de gamma ray y densidad-neutrón siguiendo la metodología propuesta por Schlumberger (1996) y Kennedy (2015).

Posterior a esto, se determinaron los intervalos de Net Rock siguiendo la metodología de Cobb y Marek, (1998). Se calculó la porosidad para los niveles siliciclásticos utilizando el registro de densidad. Para el cálculo de saturación, primero se calculó el Factor de formación $(F)$ utilizando la ecuación de Archie (Archie, 1941), se calculó la resistividad del agua $(R w)$ utilizando un pickett plot de resistividad vs porosidad como lo sugiere Pickett, (1973). También se calculó la saturación de agua utilizando el método de Doble Agua 
(DW) además de la ecuación de Archie. Antes de hacer los cálculos petrofísicos, se generó un flag de calidad del hueco para todos los ciclos de interés. El objetivo de generar un flag es determinar los intervalos en donde las condiciones del diametro del hueco no son buenas, es decir, zonas en donde pueden haber derrumbes de las paredes del pozo, por lo tanto las lecturas de las herramientas no son confiables (especialmente las herramientas de densidad y neutrón). Los intervalos seleccionados usan el registro de CALIPER y DRHO Merged. Se utilizó un cutoff para el diametro del hueco entre 8,5 y 9,25 pulgadas y un cutoff para la correción de densidad (DRHO) de $0 \mathrm{y} \pm 0,1 \mathrm{~g} / \mathrm{cc}$, estas herramientas van pegadas a las paredes del pozo, cuando existen derrumbes ellas quedan lejos de la pared del pozo y por eso se producen las lecturas erróneas (Schlumberger, 1996), por tal motivo se descartaron las lecturas que no pertenecieran a los rangos mencionados.

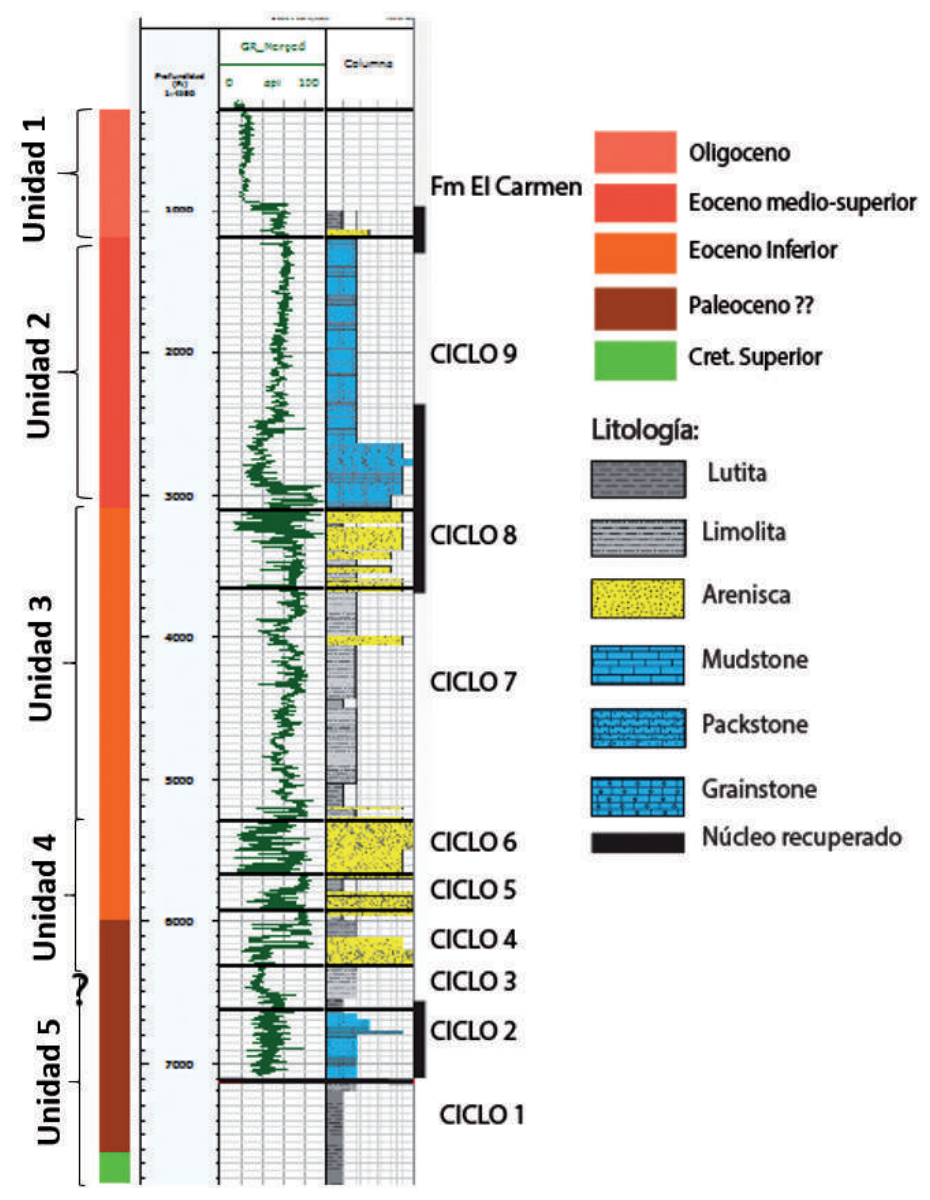

Figura 3. División del pozo ANH-TIERRALTA-2-X-P en ciclos y unidades basándose en la curva de gamma ray y la litología descrita. Se observan los intervalos (recuadro negro) con recuperación de núcleo durante la perforación.

\section{RESULTADOS}

\section{Resultados petrográficos}

El análisis petrográfico se hizo en el Ciclo 8 (Unidad 3) $(3107 \mathrm{ft}-3650 \mathrm{ft})$ ya que este fue el único intervalo siliciclástico con recuperación de núcleo. Las muestras analizadas, corresponden a cuarzoarenitas de grano fino a grueso, sublitoarenitas y litoarenitas (Figura 4) moderadas a bien seleccionadas.
Mineralógicamente las rocas están compuestas por cuarzo monocristalino $(\mathrm{Qm})$, feldespato potásico $(\mathrm{FK})$, plagioclasas $(\mathrm{Pl})$, fragmentos líticos de lutita $(\mathrm{Lu})$, cuarcíticos $(\mathrm{LmQ})$, chert $(\mathrm{Ch})$ y en menor proporción líticos volcánicos $(\mathrm{LV})$ y fragmentos de esquisto $(\mathrm{LmS})$.

Cuarzo: El Qm se presenta en todas las rocas, en porcentajes hasta en un $65 \%$, el cuarzo policristalino (Qp) se presenta en porcentajes bajos hasta en un 14\% y está ausente en algunas rocas (Figuras 5 y 6). 


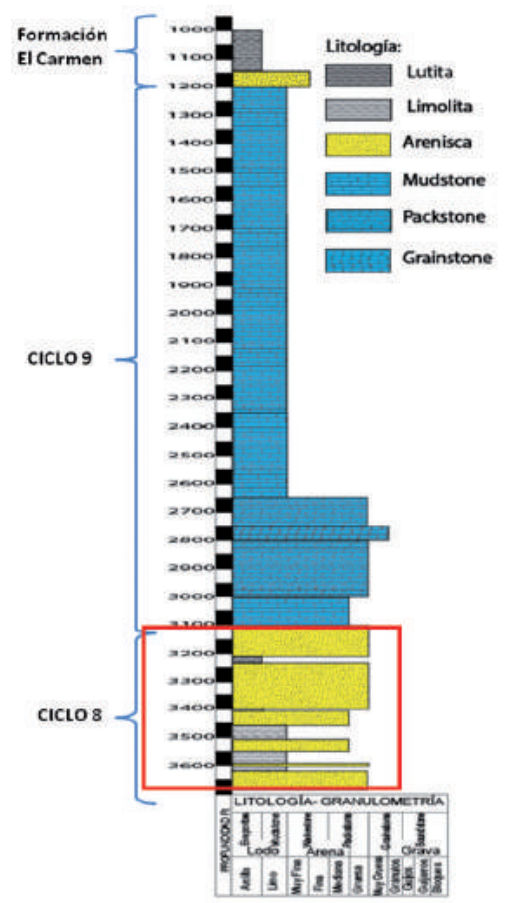

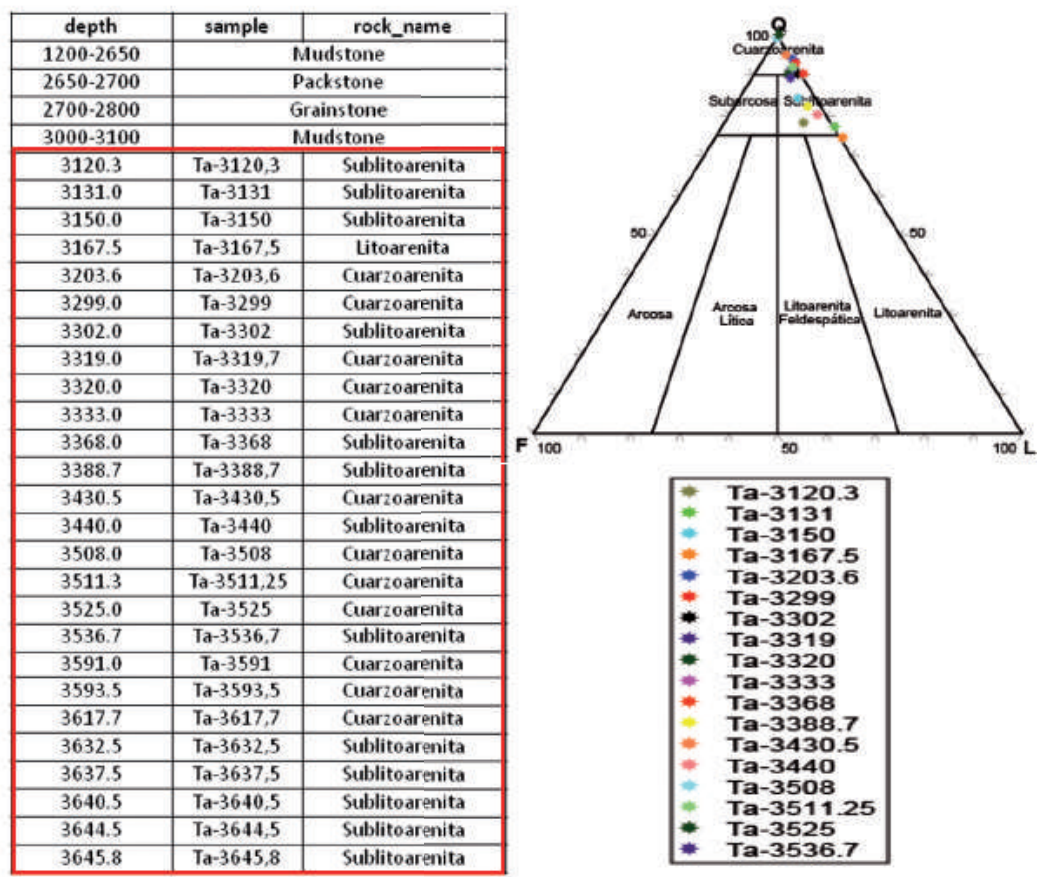

Figura 4. Clasificación composicional y ubicación en profundidad de las muestras analizadas para el Ciclo 8 siguiendo la propuesta de Folk (1974). Las muestras se encuentran ubicadas en el campo de cuarzoarenita, sublitoarenita y litoarenita.
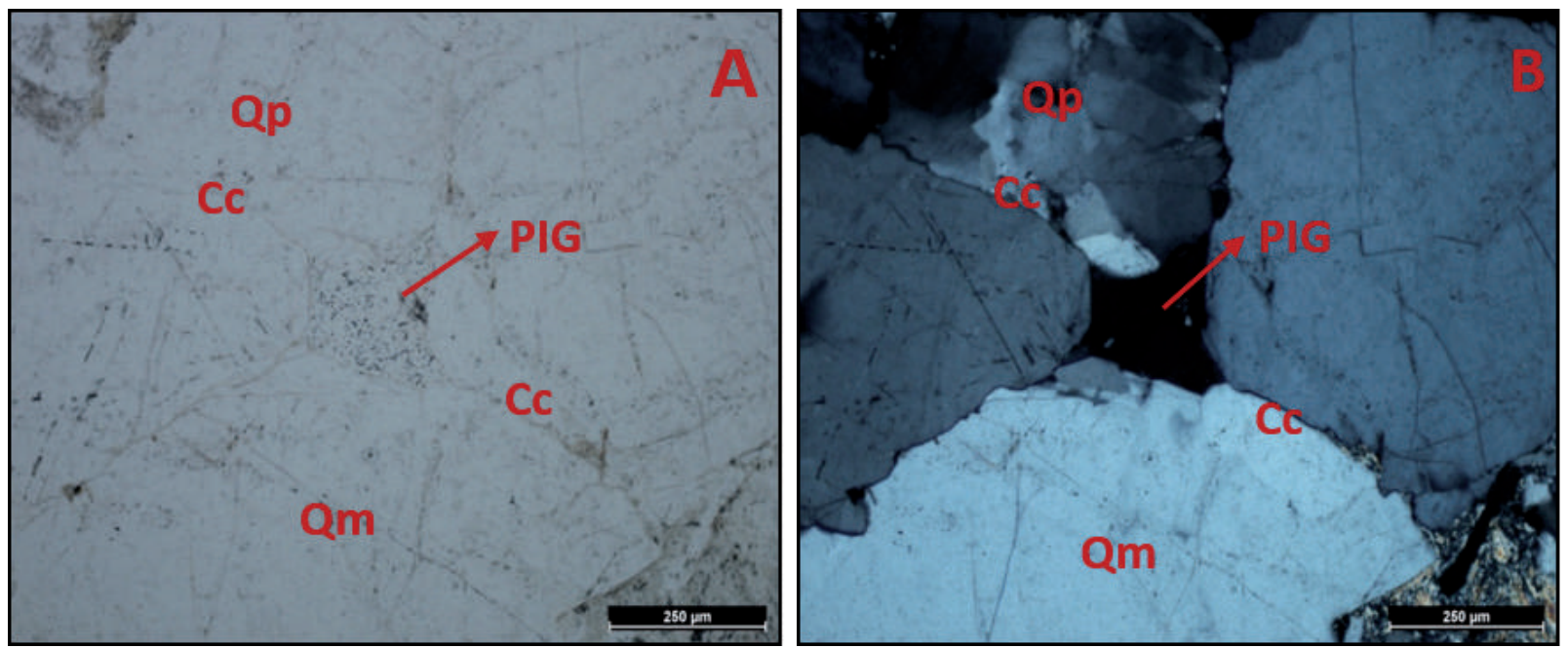

Figura 5. Sublitoarenita en A. luz polarizada plana y B. nicoles cruzados. Se observa porosidad primaria intergranular (PIG), contactos cóncavo-convexos (Cc) entre cuarzo monocristalino (Qm) y cuarzo policristalino (Qp). Muestra Ta-3120.3. 

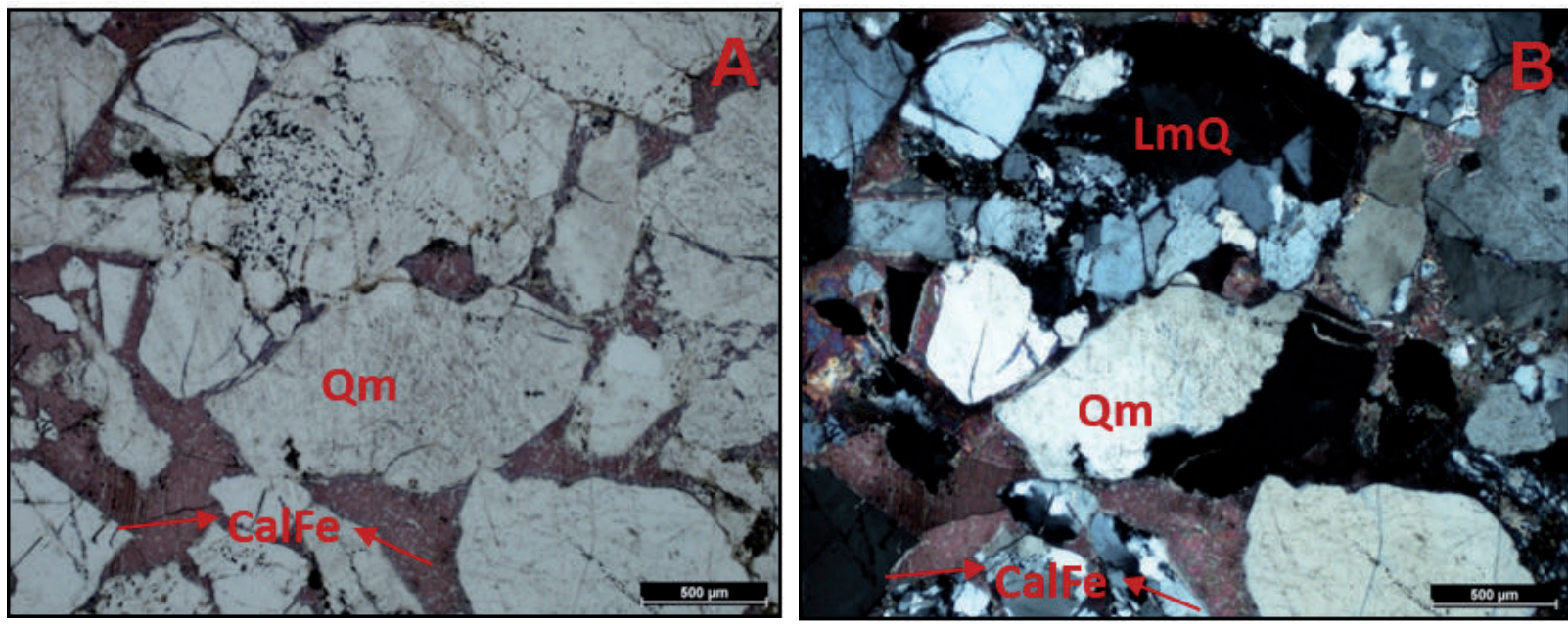

Figura 6. Litoarenita en A. luz polarizada plana y B. nicoles cruzados, el cemento de poros esparítico de calcita ferrosa (CalFe) aparece rellenando fracturas y los espacios intersticiales, clastos de cuarzo monocristalino (Qm) y cuarcita (LmQ). Muestra Ta3167.5.

Feldespatos: Los feldespatos alcalinos y plagioclasas se presentan en porcentajes bajos, los FK alcanzan hasta un $2 \%$ y corresponden a ortoclasas y microclina; las plagioclasas alcanzan un 2,5\%, aunque están ausentes en algunas muestras.

Fragmentos líticos: los fragmentos líticos abundantes corresponden a líticos sedimentarios de lutitas $(\mathrm{Lu})$ hasta un $8 \%$ y en algunas muestras se presenta chert (Ch) hasta un 4\%, fragmentos líticos metamórficos de cuarcita (LmQ) hasta un 7\%, líticos plutónicos (LPt) de granito hasta un 2\%, líticos volcánicos (LV) alterados comúnmente a clorita hasta un $1 \%$. Algunas muestras no presentan ninguno de estos componentes (Figuras 5 y 6 ).

Otros: los minerales accesorios no son muy abundantes, alcanzando hasta un $11 \%$ en algunas muestras, en orden de abundancia predominan las micas (biotita, moscovita y clorita) con un $4 \%$, pirita $1,4 \%$, siderita $3,8 \%$, caolinita $2,2 \%$, entre los minerales densos se tiene glauconita $0,2 \%$, esfena $0,1 \%$ y turmalina $0,2 \%$.

\section{Procesos y productos diagenéticos}

Se identificaron principalmente: compactación, neoformaciones minerales y metasomatismos diagenéticos. La compactación es evidenciada por los contactos cóncavo-convexos entre los granos y las deformaciones mecánicas de las micas (Figura 5) y los clastos de lutitas. Las neoformaciones minerales están representadas por el cemento carbonatado de poros y en menor proporción silíceo y caolinita. Los cementos carbonatados se presentan en la mayoría de las muestras analizadas para el Ciclo 8, sin mostrar ninguna tendencia preferencial. Su porcentaje oscila entre $0,3 \%$ y $22,7 \%$ y se encuentran asociados con la porosidad y rellenando las fracturas, en general está constituido por cemento esparítico (Figura 6). Los metasomatismos son de fragmentos líticos, cuarzo por carbonato, feldespato por caolín, matriz arcillosa por siderita y plagioclasa por sericita.

\section{Secuencia paragenética y relación con las etapas diagenéticas}

La historia diagenética que se plantea para el Ciclo 8 se estableció en términos relativos de tiempo siguiendo la metodología establecida por Worden y Burley (2003). Los criterios seguidos para este ciclo son los propuestos por Burley (1984). La síntesis de la evolución diagenética para el Ciclo 8 se presenta en la Figura 7.

El primer proceso que se presentó fue probablemente la compactación, la cual está representada por los contactos cóncavo-convexos entre los granos y las deformaciones mecánicas de las micas y algunos fragmentos de lutita. Los cementos probablemente están relacionados con la eogénesis, aunque también se pueden desarrollar en la mesogénesis hasta llegar a la telogénesis. La primera generación corresponde probablemente a los cementos de poros compuestos de sílice, asociados con la porosidad primaria, posteriormente se desarrollan los procesos de corrosión que afectan los granos de cuarzo, feldespato y fragmentos líticos, lo cual puede estar relacionado 
con la diagénesis de enterramiento (mesogénesis). Esto da inicio a los procesos de metasomatismo que afectan dichos granos. La caolinita ocurre como cemento en la etapa eogenética o telogenética por la interacción del sedimento con aguas meteóricas, esto puede ocurrir como producto de la alteración de feldespato potásico en la etapa mesogenética (Worden y Burley, 2003).

\begin{tabular}{|c|c|c|}
\hline Procesos diagenéticos & Diagénesis temprana & Diagénesis tardia \\
\hline Deformación mecánica de micas & & \\
\hline \multicolumn{3}{|l|}{ Contactos cóncavo-convexos } \\
\hline Cemento siliceo de poros & -------" & \\
\hline \multicolumn{3}{|l|}{ Cemento de carbonato de poros } \\
\hline Cemento de caolinita & -------- & \\
\hline $\begin{array}{l}\text { Metasomatismo de feldespato por } \\
\text { caolin }\end{array}$ & ????????? & \\
\hline $\begin{array}{l}\text { Metasomatismo de plagioclasa por } \\
\text { sericita }\end{array}$ & ????????? & \\
\hline \multicolumn{3}{|l|}{ Porosidad por disolución } \\
\hline \multicolumn{3}{|l|}{$\begin{array}{l}\text { Neoformismo agradante del cemento } \\
\text { carbonatado }\end{array}$} \\
\hline Muy importante & 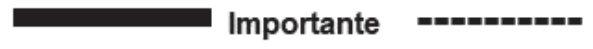 & portante \\
\hline
\end{tabular}

Figura 7. Secuencia paragenética para el Ciclo 8 del pozo ANH-TIERRALTA-2-X-P. Los principales eventos diagenéticos se presentaron durante la diagénesis temprana y diagénesis tardía.

El neomorfismo se observa en los cristales desarrollados de esparita asociados a cemento carbonatado (Figura 6). Posterior a los cementos se presenta el proceso de disolución de los granos, este se relaciona con la etapa de telogénesis en donde los fluidos meteóricos interactúan con los diferentes componentes (granos, matriz arcillosa y cemento carbonatado) llevando a la formación de porosidad secundaria, lastimosamente, para este ciclo se observó muy baja porosidad secundaria.

\section{Resultados petrofísicos}

\section{Cálculo de volumen de shale ( $\left.V_{\text {sh }}\right)$}

El volumen de shale se calculó utilizando los registros de gamma ray y densidad-neutrón, con el registro de gamma ray se aplicó el Método Lineal y la fórmula (Glover, 2000):

$$
V_{s h}=\frac{G r_{l o g}-G r_{m a}}{G r_{s h}-G r_{m a}}
$$

Donde, $V_{s h}$ es el volumen de shale en la formación; $G r_{\log }$ es la lectura del registro de gamma ray $(G r)$ en la zona de interés, en unidades API; $G r_{m a}$ es la lectura del registro de $G r$ en zonas de matriz limpia, en unidades API; $G r_{s h}$ es la lectura del registro de $G r$ en lutitas o shales, en unidades API.

Con la combinación de registros de densidad-neutrón se hizo el cálculo de volumen de shale utilizando la fórmula (Kennedy, 2015):

$$
V_{s h}=\frac{(X 1-X 0)}{(X 2-X o)}
$$

$X 0=N P H I_{M A} ; \quad X 1=N P H I_{M A}+\quad M 1^{*} \quad\left(R_{H O B}{ }_{M A}^{-}\right.$ $\left.R H O B_{M A}\right) ; X 2=N P H I_{S H}+M 1 *\left(R^{*} H O B_{M A}-R H O B_{S H}\right)$;

$M 1=\frac{N P H I_{F L}-N P H I_{M A}}{R H O B_{F L}-R H O B_{M A}}$

Donde, $\mathrm{NPHI}_{M A}$ : lectura del registro de neutrón en $100 \%$ matriz de roca; $N P H I_{F L}$ : lectura del registro de neutrón en $100 \%$ agua; $N P H I_{S H}$ : lectura del registro de neutrón en $100 \%$ shale; $R H O B_{F L}$ : lectura del registro de densidad en $100 \%$ agua; $R H O B_{M A}$ : lectura del registro de densidad en $100 \%$ matriz de roca; $R H O B_{S H}$ : lectura del registro de densidad en $100 \%$ shale. 
Cálculo de volumen de shale $\left(V_{s h}\right)$ para el Ciclo 4:

Este ciclo a pesar de no tener recuperación de núcleo, es un ciclo de interés, predomina de base a techo el contenido de areniscas conglomeráticas y areniscas cuarzosas sobre lutitas y presentó manifestaciones de hidrocarburos durante su perforación (ANH, 2014a). La Figura 8 muestra las curvas finales de $V_{s h}$ calculadas para el Ciclo 4. Para el cálculo de $V_{s h}$ se utilizó un $G r_{m a}=$
25 API y un valor de $G r_{s h}=86$ API. La Figura 9 muestra el cross plot de los registros de neutrón $v s$ densidad utilizado para determinar los valores de entrada en la ecuación 2, para este pozo se utilizaron los valores de $R H O B_{M A}=2,65 \mathrm{~g} / \mathrm{cc}$ (areniscas cuarzosas) y $\mathrm{RHOB}_{F L}=$ $1 \mathrm{~g} / \mathrm{cc}$ (lodos base agua); los demás valores se obtienen gráficamente (Spooner, 2014).

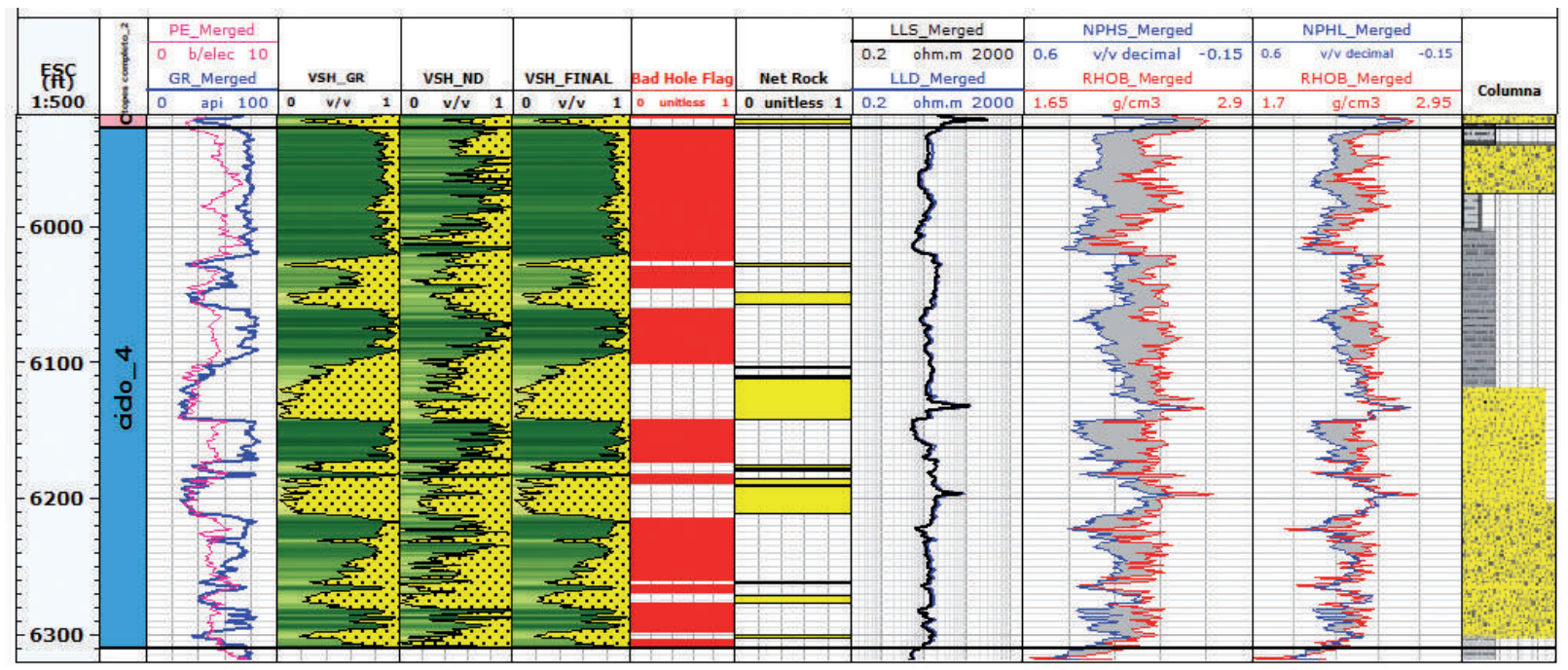

Figura 8. Cálculo de $V_{s h}$ para el Ciclo 4 a partir del registro de gamma ray (Gr) y densidad-neutrón. Se observa además la combinación de registros de densidad (RHOB) y neutrón (NPH). Los campos rojos son las zonas donde se aplicó el flag de calidad del hueco y los campos amarillos corresponden a las zonas de Net Rock.

\section{Cálculo de volumen de shale $\left(V_{s h}\right)$ para el Ciclo 5:}

Este ciclo presenta niveles de areniscas interestratificadas con lutitas grises y se reportaron manifestaciones de hidrocarburos durante su perforación (ANH, 2014a). La Figura 10 muestra las curvas finales de $V_{s h}$ calculadas para el Ciclo 5. Para el cálculo de $V_{s h}$ se utilizó un $G r_{m a}=24$ API y un valor de $G r_{s h}=86$ API.

\section{Cálculo de volumen de shale $\left(V_{s h}\right)$ para el Ciclo 6:}

Este ciclo no tiene recuperación de núcleo, pero representa un ciclo de interés ya que es siliciclástico y presentó manifestaciones de hidrocarburos durante su perforación (ANH, 2014a). La Figura 11 muestra las curvas finales de $V_{s h}$ calculadas para el Ciclo 6. Utilizando 12 unidades API como valor de $G r_{m a}$ y
84 unidades API como valor de $G r_{s h}$ y se utilizó la ecuación 1 para calcular el volumen de shale.

\section{Cálculo de volumen de shale $\left(V_{s h}\right)$ para el Ciclo 7:}

Este ciclo corresponde a lutitas, y en menor cantidad arenisca. La lutita se presenta color gris verdoso claro, gris medio; blanda, localmente limoso; amorfa, ligeramente soluble y calcárea (ANH, 2014b). La arenisca es cuarzosa de grano muy fino, los granos son subangulares a subredondeados y moderada selección, su cemento es calcáreo y presenta trazas de glauconita.

Para el cálculo del volumen de shale $\left(V_{s h}\right)$ se utilizó 25 unidades API como valor de $G r_{m a}$ y 80 unidades API como valor de $G r_{s h}$ (Figura 12). 


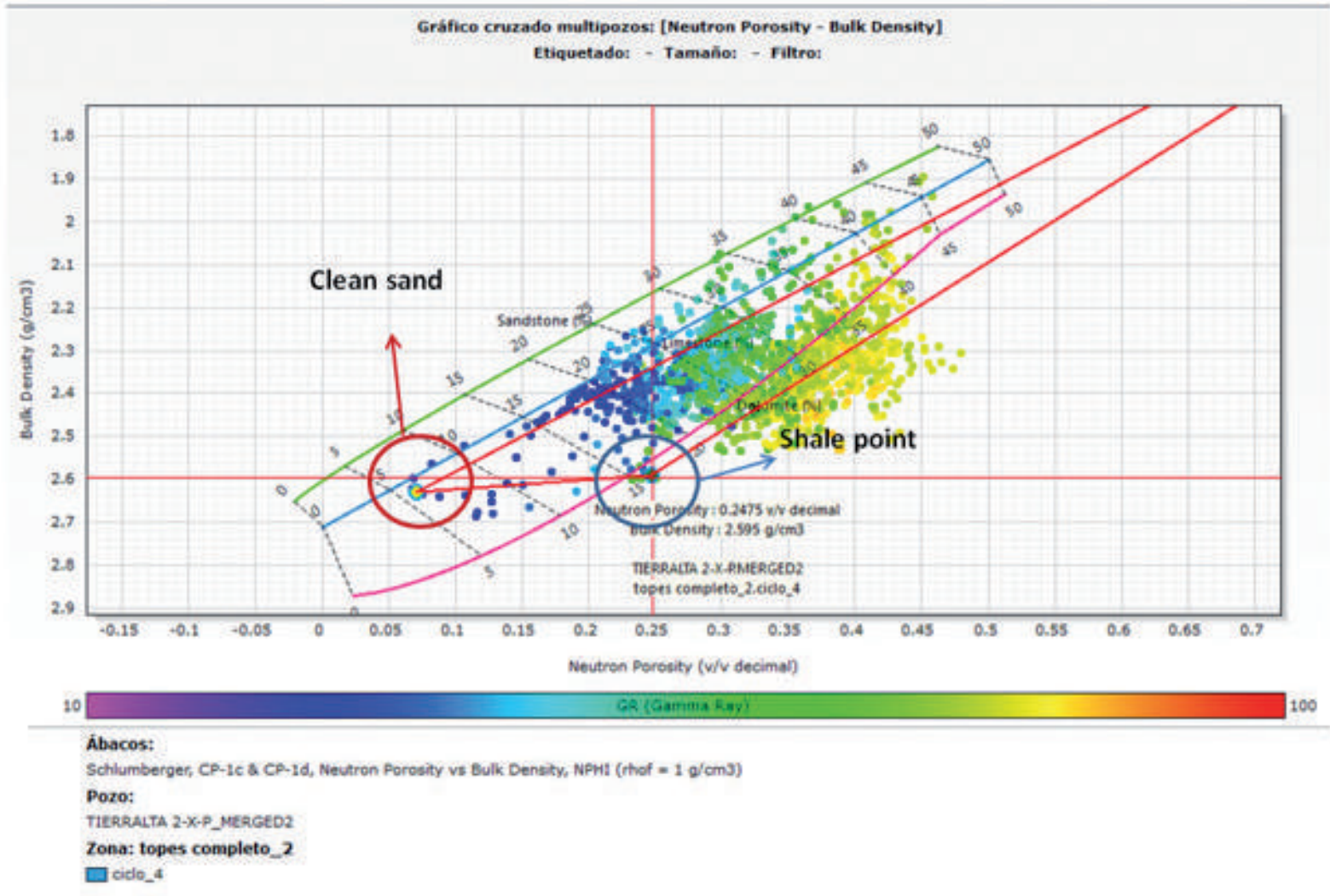

Figura 9. Cross plot de registro de densidad RHOB_Merged vs registro de neutrón. Se observan los puntos para determinar los valores de entrada para el cálculo de $V_{s h}$ a partir de los registros de densidad-neutrón (Spooner, 2014).

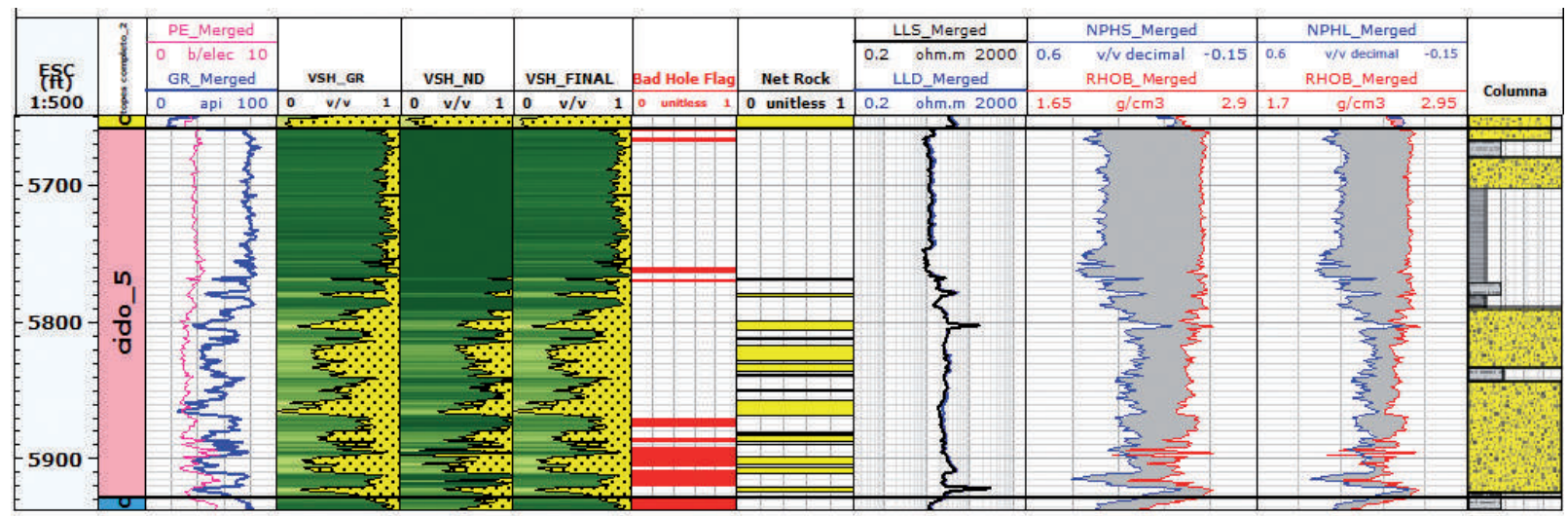

Figura 10. Cálculo de $V$ para el Ciclo 5 a partir del registro de gamma ray (Gr) y densidad-neutrón. Se observa además la combinación de registros de densidad (RHOB) y neutrón (NPH). 


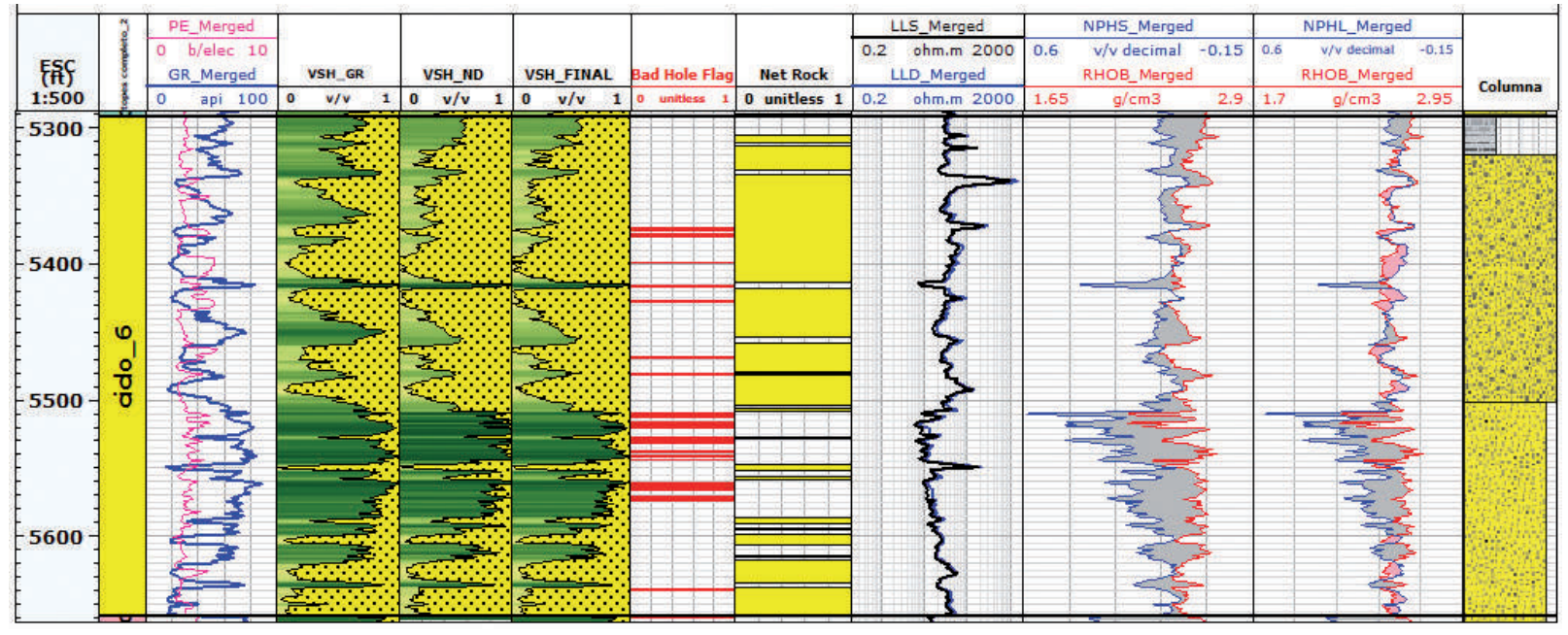

Figura 11. Cálculo de $V_{\text {sh }}$ para el Ciclo 6 a partir de gamma ray $(\mathrm{Gr})$ y densidad-neutrón. Se observa además la combinación de registros de densidad (RHOB) y neutrón (NPH).

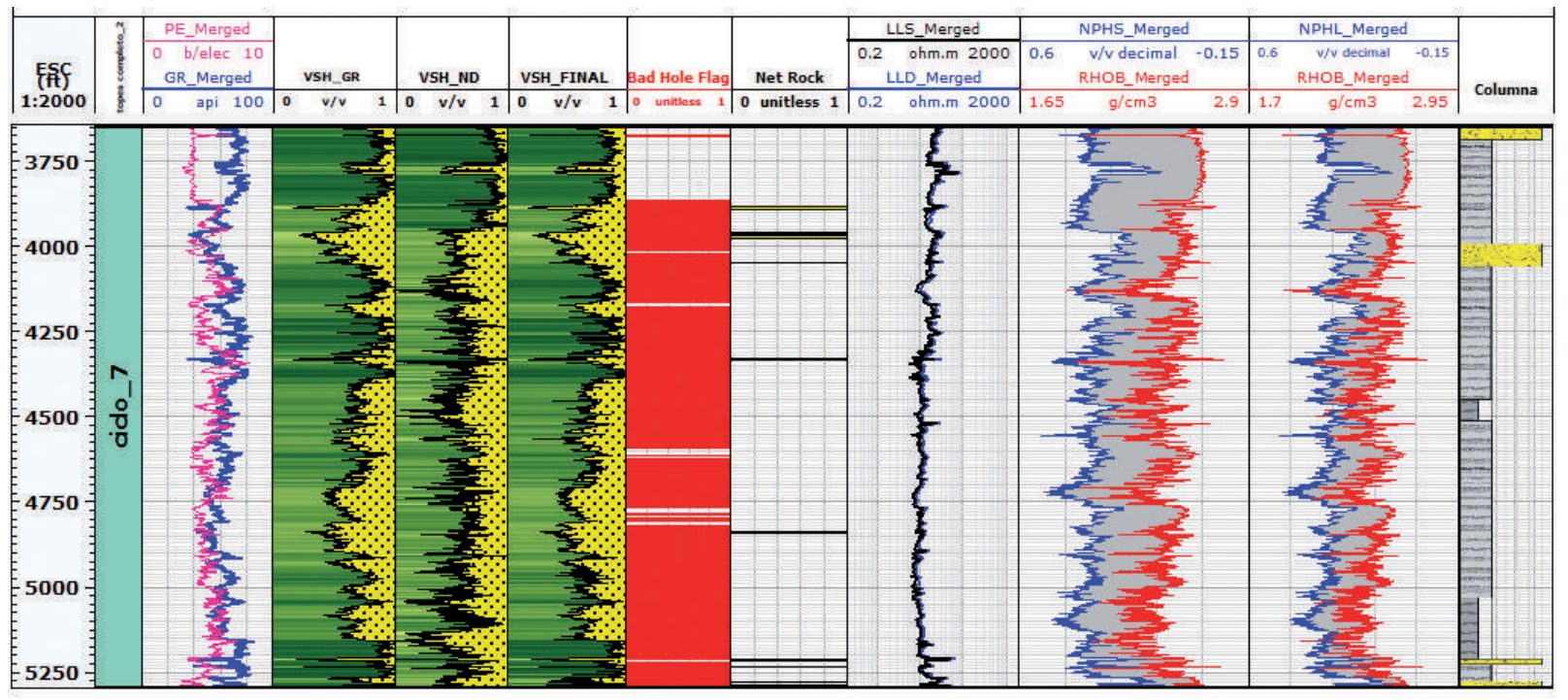

Figura 12. Curva de $V_{\text {sh }}$ para el Ciclo 7 a partir de gamma ray $(\mathrm{Gr})$ y densidad-neutrón. Se observa además la combinación de registros de densidad (RHOB) y neutrón (NPH). 
Cálculo de volumen de shale $\left(V_{s h}\right)$ para el Ciclo 8:

Este ciclo corresponde a una intercalación de cuarzoarenitas (Figuras 13 y 14) al tope con lutitas a la base; utilizando la ecuación 1 se obtiene un volumen de shale promedio que alcanza un $18,3 \%$ en las zonas de Net Rock de la formación (Figura 14), se calculó usando 8 unidades API como valor de $G r_{m a}$ y 95 unidades API como valor de $G r_{s h}$. Al hacer un ajuste registro-roca se observa que los datos de los registros concuerdan con la descripción del núcleo y de petrografía, la Figura 13 es un ejemplo claro de este ajuste, en donde la respuesta de la curva de gamma ray
(Gr), neutrón (NPH) y densidad (RHOB) indican una litología aparentemente de arenisca, como lo confirma el núcleo, además se observa que las areniscas se encuentran cementadas por carbonatos.

La Tabla 1 resume los valores de volumen de shale final para los ciclos 4, 5, 6, 7 y 8 calculados a partir de los registros de gamma ray y densidad-neutrón, además del espesor del intervalo (Gross), zonas limpias (Net Rock) y la relación espesor/zonas limpias (Net/Gross) (Schlumberger, 2013).

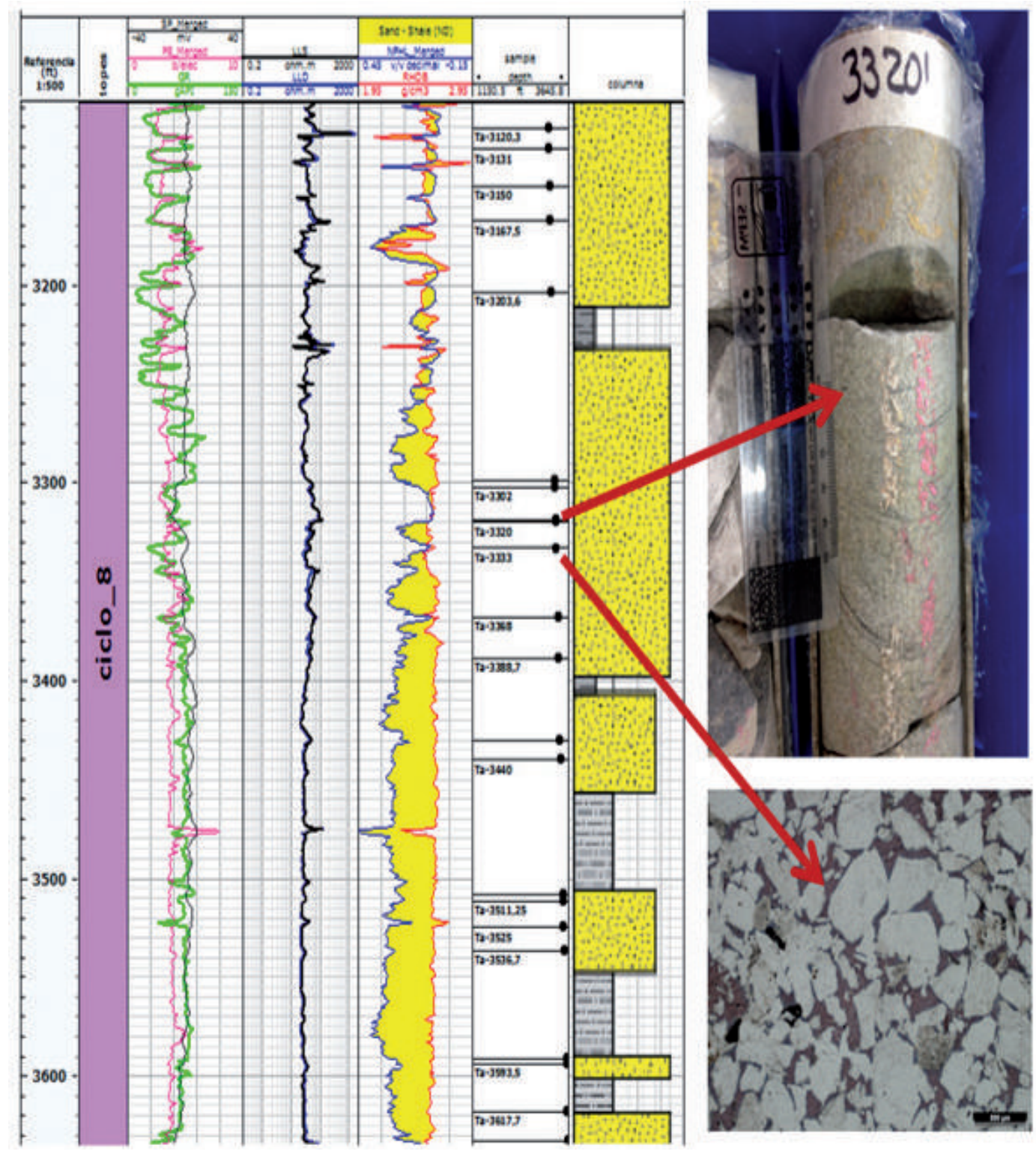

Figura 13. Amarre registro-roca para las unidades siliciclásticas del Ciclo 8. Se observa que las areniscas están cementadas por cemento calcáreo. 


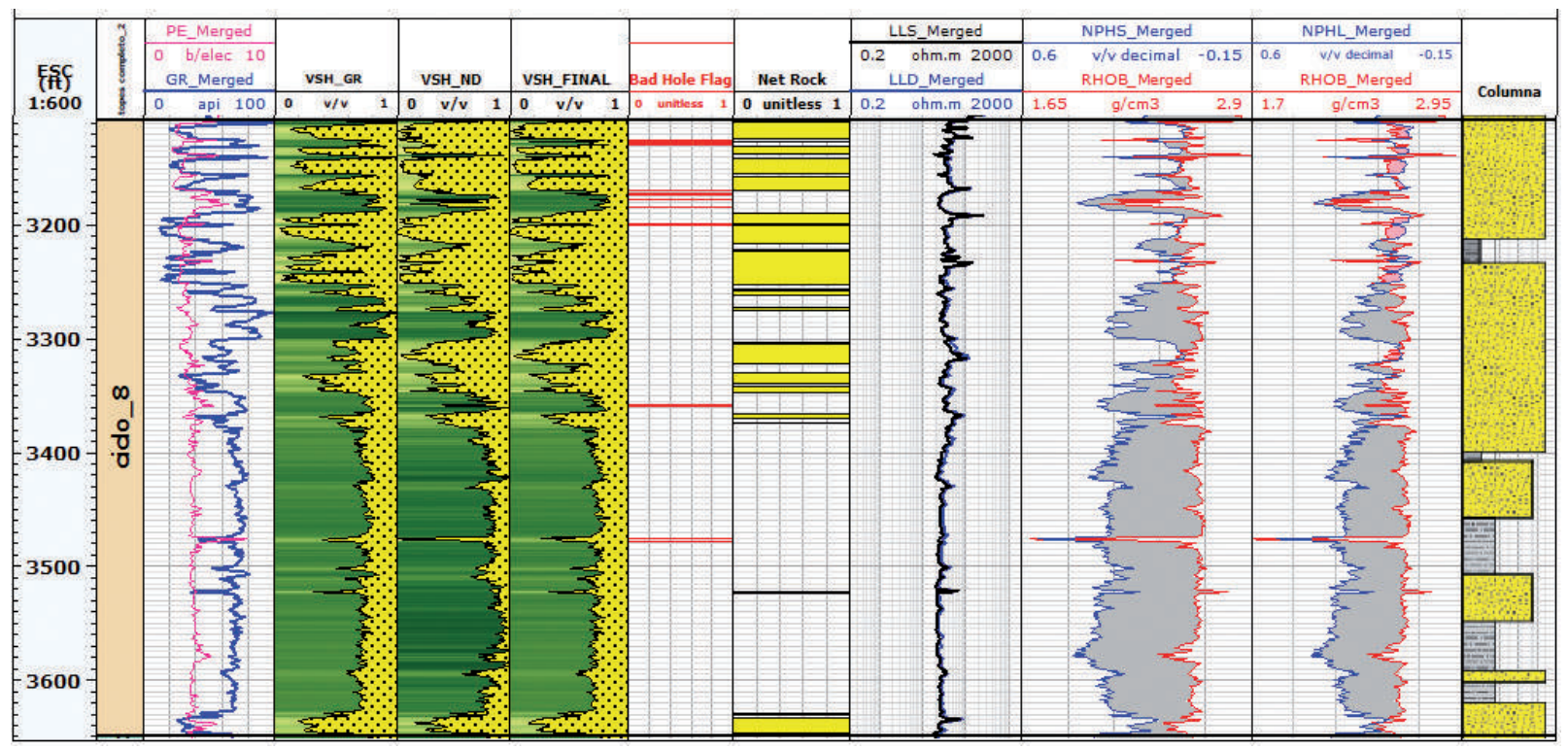

Figura 14. Curva de $V_{s h}$ para el Ciclo 8 a partir de gamma ray (GR) y densidad-neutrón. Se observa además la combinación de registros de densidad (RHOB) y Neutrón (NPH).

Tabla 1. Valores promedio de $V_{s h}$ final para los ciclos 4, 5, 6, 7 y 8 calculados a partir de los registros gamma ray y densidadneutrón en las zonas de Net Rock.

\begin{tabular}{cccccc}
\hline Ciclo & $\boldsymbol{V}_{\text {sh }}$ Final (\%) & Gross (ft) & $\begin{array}{c}\text { Net Rock } \\
(\mathbf{f t})\end{array}$ & Net/Gross & $\begin{array}{c}\text { Cutoff } \\
\mathbf{m a x}(\%)\end{array}$ \\
\hline 4 & 13,1 & 382,35 & 78,5 & 0,20 & 30 \\
5 & 31,5 & 269,28 & 61,76 & 0,22 & 45 \\
6 & 20,75 & 366,14 & 233,1 & 0,66 & 50 \\
7 & 27,6 & 164,80 & 3,65 & 0,02 & 35 \\
8 & 18,3 & 541,12 & 164,75 & 0,30 & 40 \\
\hline
\end{tabular}

\section{Cálculo de porosidad a partir del registro de densidad}

Al hacer un gráfico cruzado (cross-plot) de permeabilidad vs porosidad (Figura 15) se puede observar que no existe una correlación clara entre los valores de permeabilidad y porosidad de las muestras, esto puede presentarse debido a varios parámetros: 1) distribución del tamaño de grano; 2) distribución del tamaño del poro; 3) interconexión de los poros, y 4) la tortuosidad (vías de flujo dentro de la roca) (Buryakovsky et al., 2012). La Tabla 2 muestra los datos de porosidad y permeabilidad calculados en el laboratorio. Los registros de densidad se usan principalmente como registros de porosidad además de otros usos como la identificación de minerales y detección de gas (Schlumberger, 1990).

Una fuente radiactiva emite a la formación, rayos gamma de mediana energía que chocan con los electrones de ella; con cada choque, los rayos pierden algo de su energía; esta clase de interacción se conoce como efecto Compton. El número de colisiones en el efecto Compton está directamente relacionado con el número de electrones de la formación. En consecuencia, la respuesta de la herramienta está asociada con la densidad de la roca $b_{b}$, que a su vez depende de la densidad del material de la matriz de la roca, la porosidad de la formación y la densidad de los fluidos que rellenan los poros (Schlumberger, 1990). Para una formación limpia con una matriz de densidad conocida $p_{m a}$, que tenga una porosidad conocida $\phi$ y contenga un líquido de densidad promedio $p_{f}$, la densidad total de la formación $p_{b}$ será:

$$
\phi=\frac{\mathrm{p}_{m a}-\mathrm{p}_{b}}{\mathrm{p}_{m a}-\mathrm{p}_{f}}
$$

\section{Cálculo de porosidad $(\phi)$ para el Ciclo 8:}

El cálculo de porosidad a partir del registro de densidad RHOB_Merged para este ciclo se hizo utilizando los valores de $b_{m a}=2,65 \mathrm{~g} / \mathrm{cc}$ por ser casi en su totalidad areniscas cuarzosas, como se mencionó anteriormente 
(no se utilizaron los datos de laboratorio debido a que los valores de densidad de grano reportados son bastante bajos en relación con los esperados para areniscas) y $p_{f}=1,0 \mathrm{~g} / \mathrm{cc}$ (lectura del registro de densidad en $100 \%$ agua). La Figura 16 muestra la curva de porosidad generada para este ciclo.

\section{Cálculo de porosidad $(\phi)$ en los Ciclos 4,5 y 6 :}

El cálculo de porosidad para estos ciclos se hizo utilizando el registro de densidad RHOB Merged y los mismos valores usados en los cálculos del Ciclo $8\left(p_{m a}\right.$ $=2,65 \mathrm{~g} / \mathrm{cc}$ y $p_{f}=1,0 \mathrm{~g} / \mathrm{cc}$ ) debido a que no se tienen datos de laboratorio de estos ciclos y la información de las descripciones litológicas hechas durante la perforación del pozo son similares a la litología descrita para el Ciclo 8. La Figura 17 muestra las curvas de porosidad generadas para estos ciclos. La Tabla 3 muestra los resultados de porosidad promedio calculados en las zonas de Net Rock.

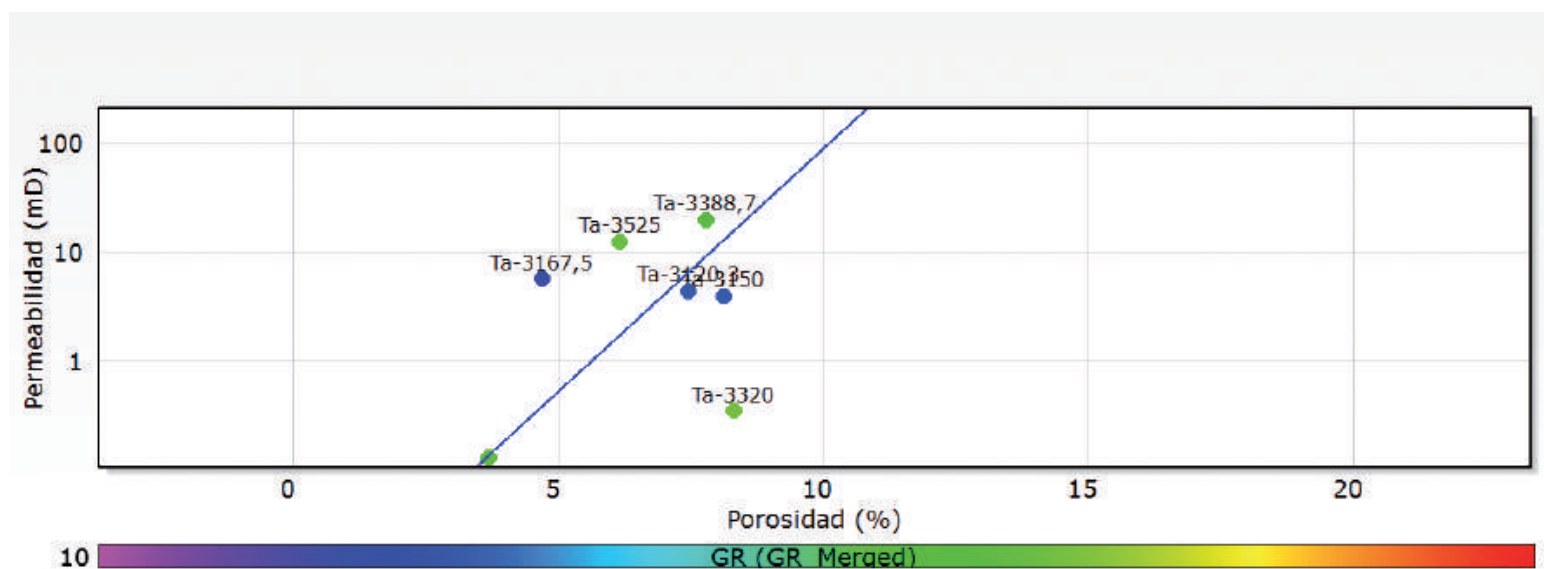

\section{Scale:}

Scale 1: [Porosidad - pemeabilidad]

Regressions:

Reg1 (type = RMA $Y / X ; R=0.327 ; R 2$ adj $=-0.072 ; R M S E=1.02432 ; n b=7$ )

Equation: $\log 10($ permeabilty $)=+0.445699 *$ Porosidad -2.507102

Figura 15. Gráfico cruzado de permeabilidad vs porosidad para el Ciclo 8. El color de cada punto representa su valor en el registro de gamma ray.

Tabla 2. Resultados de porosidad y permeabilidad analizados en el laboratorio para el pozo ANH-TIERRALTA-2-X-P.

\begin{tabular}{cccccc}
\hline $\begin{array}{c}\text { Prof. } \\
\text { (ft) }\end{array}$ & $\begin{array}{c}\text { Porosidad } \\
\mathbf{( \% )}\end{array}$ & Permeabilidad mD & Muestra & $\begin{array}{c}\text { Densidad de } \\
\text { grano (g/cc) }\end{array}$ & Observaciones \\
\hline 3120,30 & 7,46 & 4,40 & Ta-3120,3 & 2,60 & $\mathrm{n} / \mathrm{a}$ \\
3131,00 & 8,26 & 0,00 & Ta-3131 & 2,60 & Fracturada \\
3150,00 & 8,12 & 4,00 & Ta-3150 & 2,62 & $\mathrm{n} / \mathrm{a}$ \\
3167,50 & 4,69 & 5,70 & Ta-3167,5 & 2,60 & $\mathrm{n} / \mathrm{a}$ \\
3203,60 & 10,88 & 0,00 & Ta-3203,6 & 2,60 & $<0,01 \mathrm{md}$ \\
3320,00 & 8,31 & 0,35 & Ta-3320 & $\mathrm{n} / \mathrm{a}$ & $\mathrm{n} / \mathrm{a}$ \\
3333,00 & 2,35 & 0,00 & Ta-3333 & 2,63 & $<0,01 \mathrm{md}$ \\
3367,00 & 3,70 & 0,13 & Ta-3368 & $\mathrm{n} / \mathrm{a}$ & $\mathrm{n} / \mathrm{a}$ \\
3388,70 & 7,78 & 19,67 & Ta-3388,7 & $\mathrm{n} / \mathrm{a}$ & Fracturada \\
3440,00 & 0,00 & 0,00 & Ta-3440 & $\mathrm{n} / \mathrm{a}$ & no determinada \\
3508,00 & 4,95 & 0,00 & Ta-3508 & 2,64 & $<0,01 \mathrm{md}$ \\
3525,00 & 6,17 & 12,64 & Ta-3525 & $\mathrm{n} / \mathrm{a}$ & Fracturada \\
3617,50 & 0,00 & 0,00 & Ta-3617,7 & $\mathrm{n} / \mathrm{a}$ & no determinada \\
3645,80 & 8,79 & 0,00 & Ta-3645,8 & 2,60 & $<0,01$ \\
\hline
\end{tabular}




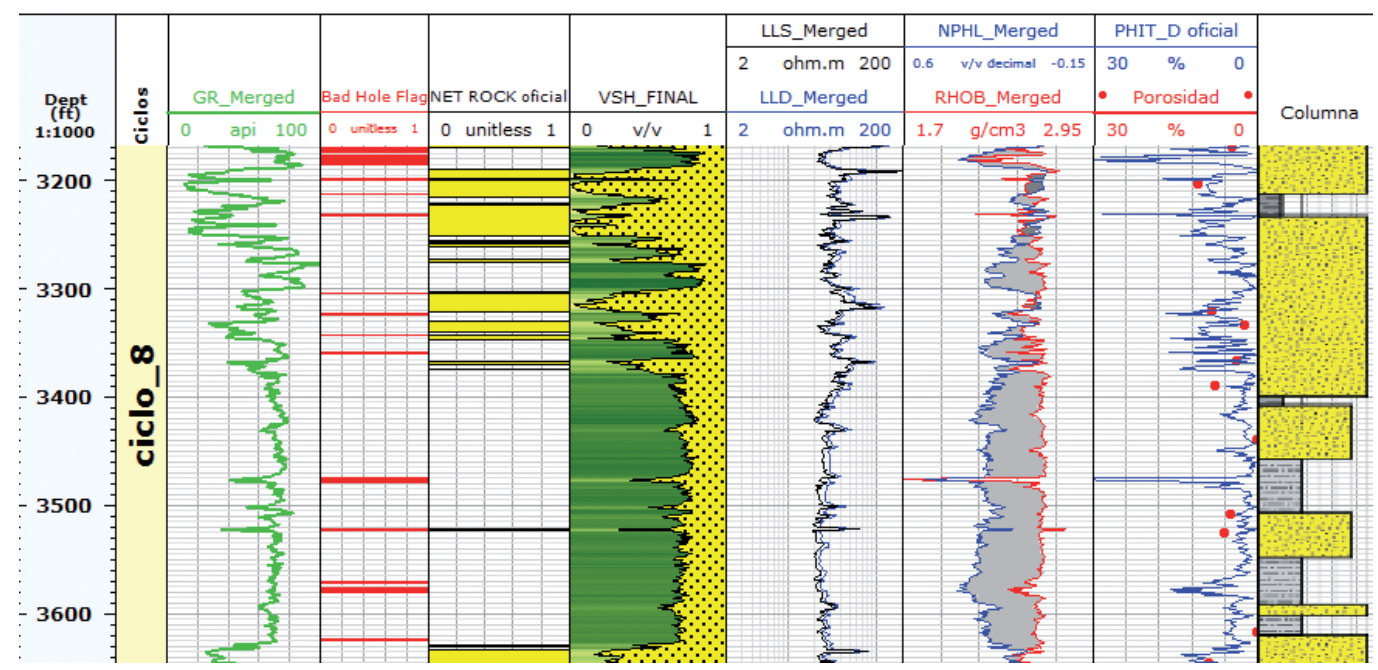

Figura 16. Registro de porosidad calculada (PHIT_D oficial) a partir del registro de densidad (RHOB_Merged) en las zonas de Net Rock para el Ciclo 8. Los puntos rojos son los valores de porosidad calculados en el laboratorio.

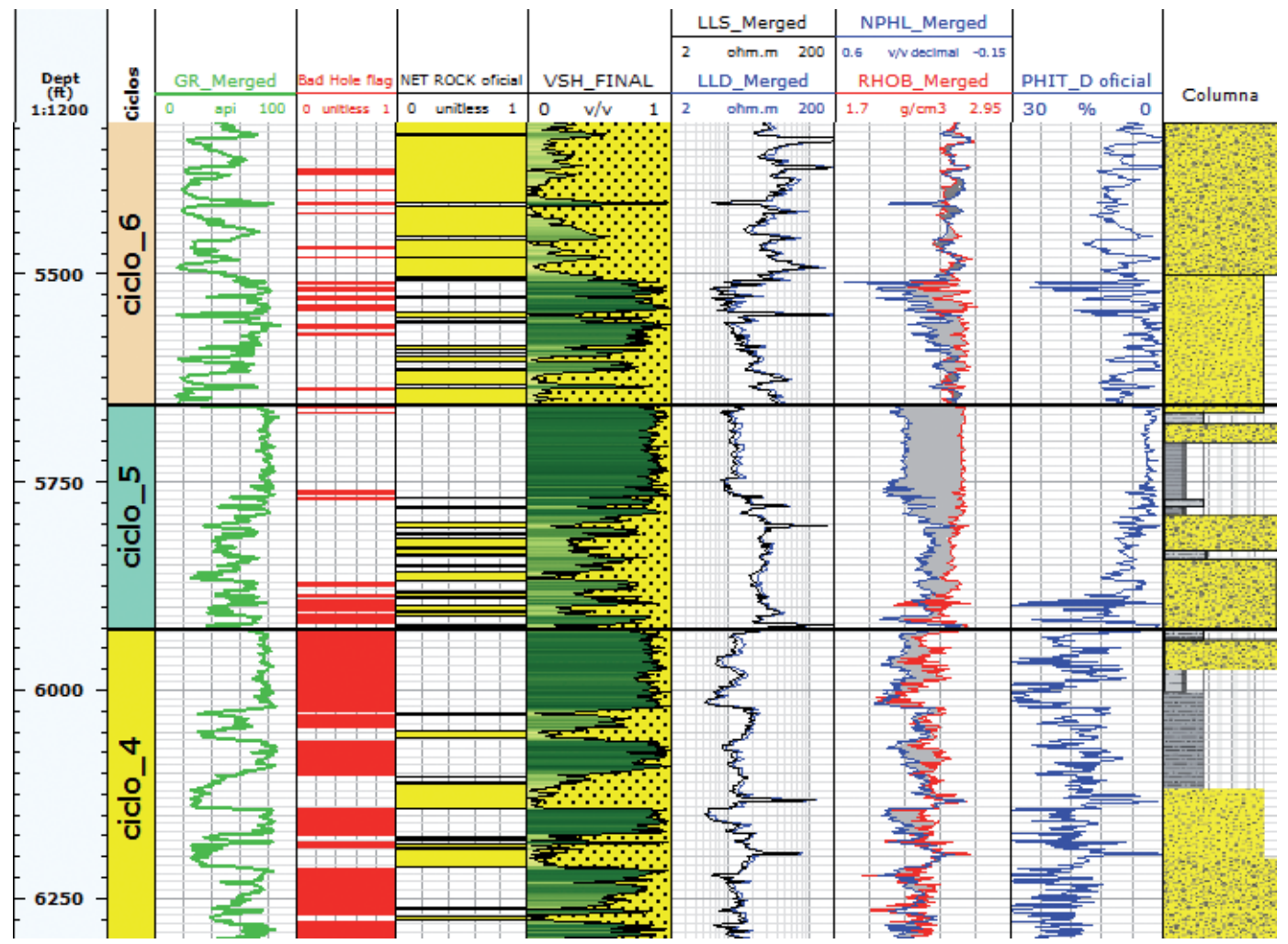

Figura 17. Registro de porosidad calculada (PHIT_D oficial) a partir del registro de densidad (RHOB_Merged) para los ciclos 4 y 5.

Tabla 3. Valores promedio de porosidad para los ciclos 4, 5, 6 y 8 calculados a partir del registro de densidad RHOB_Merged.

\begin{tabular}{cc}
\hline Ciclo & $\begin{array}{c}\text { Porosidad }(\%) \\
\mathrm{p}_{m a}=2,65 \mathrm{~g} / \mathrm{cc} \mathrm{y} \mathrm{p}_{f}=1,0 \mathrm{~g} / \mathrm{cc}\end{array}$ \\
\hline 4 & 15,1 \\
5 & 8,2 \\
6 & 8,0 \\
8 & 7,3 \\
\hline
\end{tabular}




\section{Cálculo de saturación de agua (Sw)}

Para el cálculo de saturación de agua $(S w)$, se calcularon primero los valores del factor de formación $(F)$ utilizando la ecuación 4:

$$
\mathrm{F}=\frac{a}{\phi^{m}}
$$

Donde $a=1$ (factor de tortuosidad en la zona de interés); $m=2$ (índice de cementación) y $\phi=$ porosidad calculada a partir del registro de densidad. El índice de cementación describe el incremento en la resistividad que resulta de los granos de un mineral aislante forzando la corriente eléctrica a tomar tortuosas vías a través del fluido conductivo. La ecuación 4 es aplicable en formaciones compactadas, por ejemplo, calizas y areniscas bien cementadas (Glover, 2000).

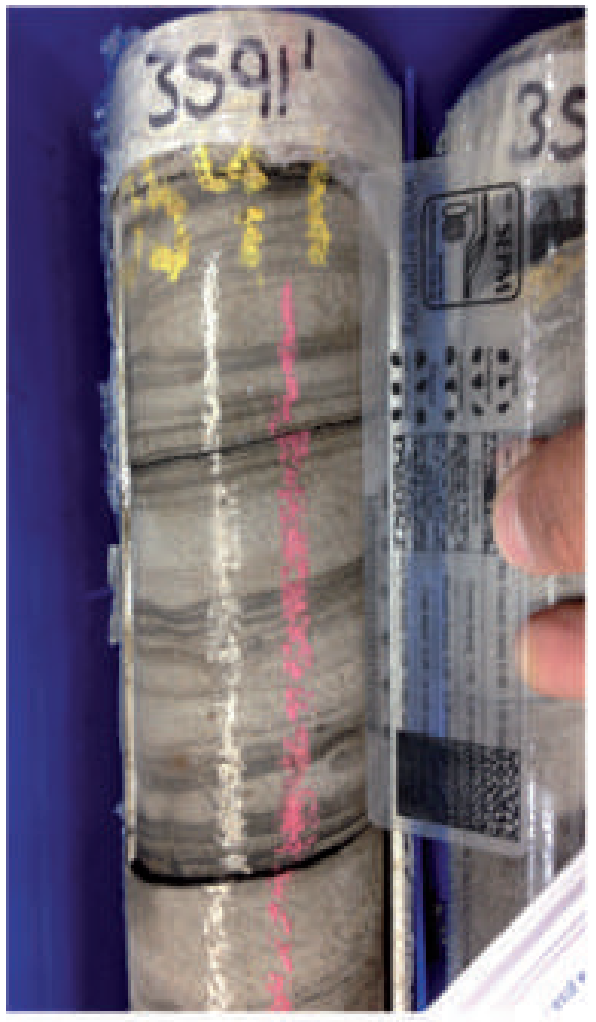

Shale laminar

La saturación de agua se calculó utilizando la ecuación de Archie (Viro, 1997) para formaciones limpias:

$$
S w=\sqrt[n]{F * \frac{R w}{R t}}
$$

Donde $R t$ es la resistividad de la formación y $R w$ es la resistividad del agua de formación, utilizando el método del pickett plott (Pickett, 1973). La Figura 18 es un ejemplo claro de las diferentes distribuciones de shale (Spooner, 2014) encontradas en el Ciclo 8. Se observa claramente que la presencia de shale puede afectar los resultados en el cálculo de saturación utilizando la ecuación de Archie (Viro, 1997) por lo tanto se calculó el $S w$ utilizando el modelo de Doble Agua (DW) (Figura 18) utilizando la ecuación 6.

$$
\begin{gathered}
\frac{a}{R t * \phi_{t}^{m}}=\frac{1}{R w} * S_{w}^{n}+Q_{V} *\left(\frac{1}{\phi_{t s h}^{2}}-\frac{1}{R w}\right) * S w^{(n-1)} \\
Q_{V}=\frac{\phi_{t s h} * \mathrm{~V}_{s h}}{\phi}
\end{gathered}
$$
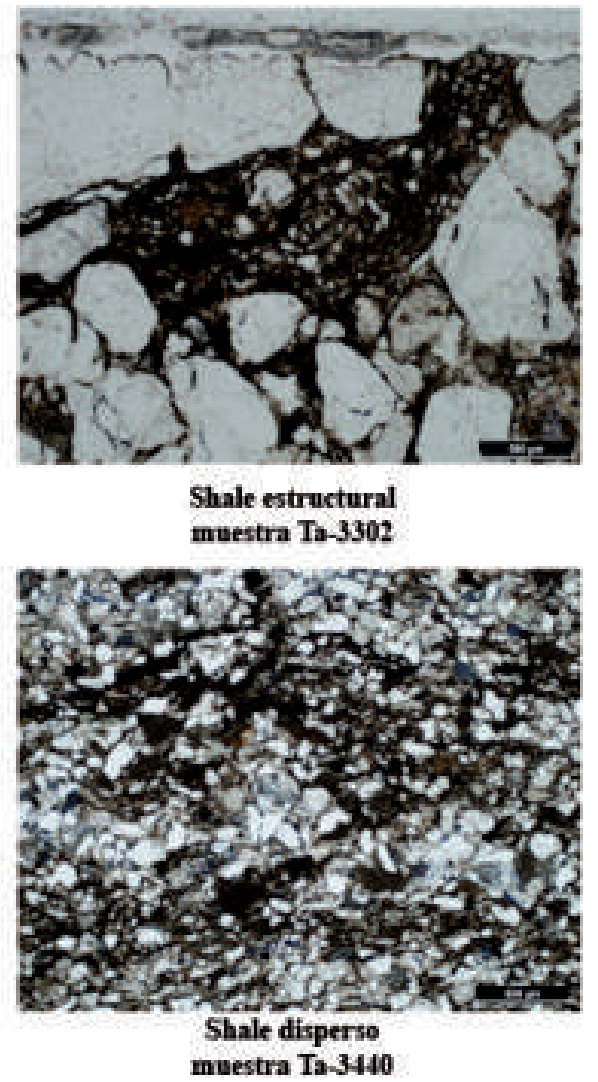

Figura 18. Distribuciones de shale encontradas en el Ciclo 8. Se observa la presencia de A. Shale laminar. B. Shale estructural como clastos de lutita. C. Shale disperso representado como matriz arcillosa rellenando los espacios intersticiales de la roca. 
Donde $R t$ es la resistividad de la formación; $R s h$ es el valor de la resistividad leída en zonas de $100 \%$ shale; $\phi_{t}$ es la porosidad total (calculada por cualquier método); $V_{s h}$ es el volumen de shale calculado por cualquier método; $a$ es el factor de tortuosidad en la zona de interés; $m$ es el factor de cementación en la zona de interés; $n$ es el exponente de saturación en la zona de interés; $R w$ es la resistividad del agua de formación en la zona de interés; $Q v$ es la capacidad de intercambio catiónico de las arcillas; $\phi_{t s h}$ es la porosidad leida en zonas de $100 \%$ shale.

Para consideraciones petrofísicas la formación arcillosa es caraterizada por su porosidad $\phi_{t}$; su factor de formación $F$; su saturación de agua $S w$ y su capacidad de intercambio catiónico convertido en unidad de volúmen $Q_{v}$. En el modelo de Doble Agua se considera que la arcilla es de dos componentes: agua ligada y minerales de arcilla (Schlumberger, 1990). Los minerales de arcilla se consideran electricamente inertes, la conductividad del agua de la arcilla de considera como derivada a la conductividad del agua ligada que se supone es independiente del tipo de arcilla. La cantidad de agua ligada varía según el tipo de arcilla y se supone que es mayor para las arcillas mas finas (con mayores áreas de superficie) como la montmorillonita y menor para arcillas mas gruesas como la caolinita (Schlumberger, 1990). Para el cálculo de $S w$ se determinó el valor de $R w$ haciendo un pickett plot. La Figura 19 muestra el pickett plot de porosidad calculada $v s$ resistividad profunda, con este método se obtuvo un $R w=0,9$ promedio para los cálculos de saturación de agua.

Para el cálculo por el método de Doble Agua (Sw_Dual), se escogieron los puntos con mayor porcentaje de shale y en ellos se tomó la resistividad y porosidad (Tabla 4). La Figura 20 muestra las curvas de $S w$ y Sw_Dual generadas para el Ciclo 8.

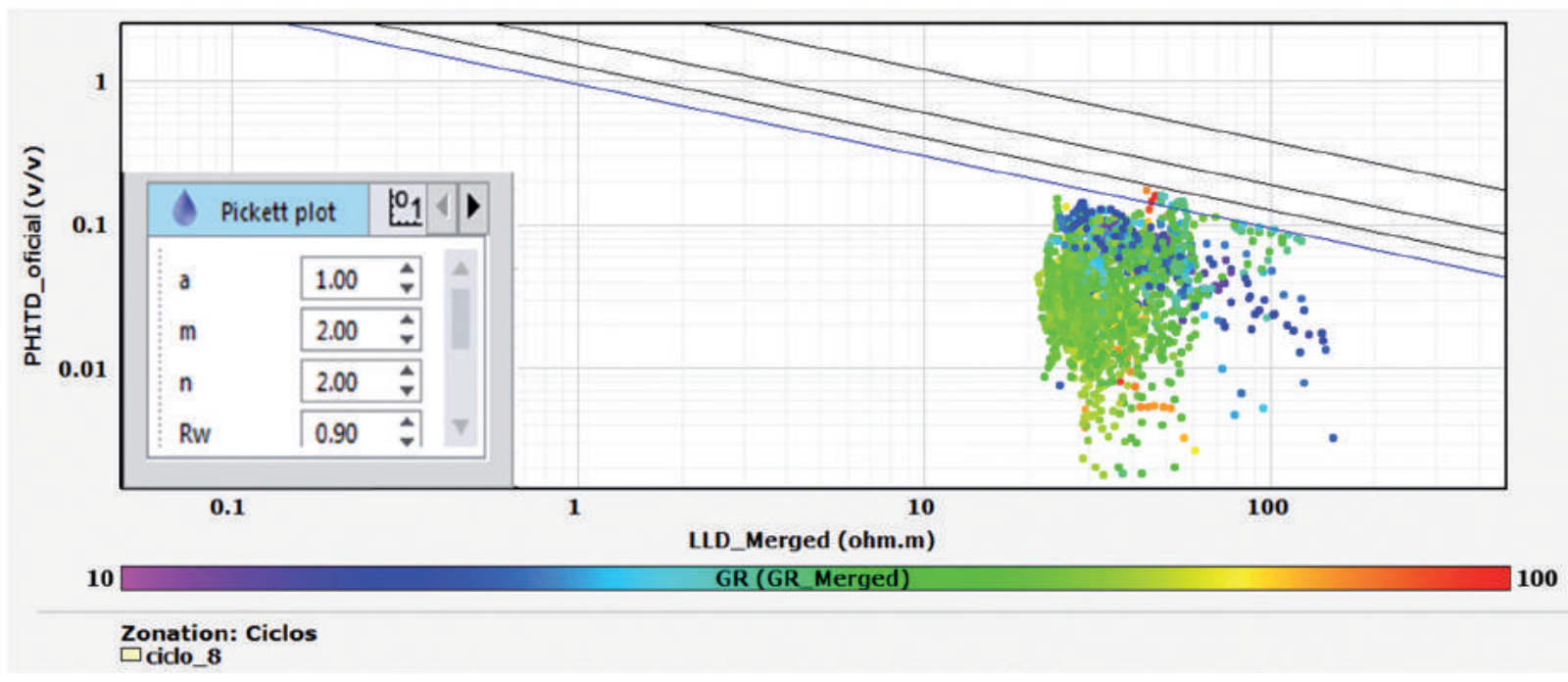

Figura 19. Picket plot porosidad (PHIID_oficial) $v s$ resistividad (LLD_Merged) profunda para la determinación de $R w=0,9$ en el Ciclo 8.

Tabla 4. Valores usados para el cálculo de saturación de agua por el método de Doble Agua (Dual water).

\begin{tabular}{cccc}
\hline Ciclo & Profundidad (ft) & $\begin{array}{c}\text { Resistividad } \\
\text { (ohm.m) }\end{array}$ & Porosidad (v/v) \\
\hline 8 & 3573,31 & 22,5 & 0,052 \\
6 & 5661,32 & 11,03 & 0,03 \\
5 & 5707,3 & 14,6 & 0,025 \\
4 & 6068 & 9,22 & 0,19 \\
\hline
\end{tabular}


Cálculo de saturación de agua $(S w)$ para el Ciclo 6:

El cálculo de saturación de agua $(S w)$ para este ciclo se hizo utilizando un $R w=0,7$ obtenido del pickett plot de porosidad $v s$ resistividad (Figura 21).

Cálculo de saturación de agua ( $S w)$ para los Ciclos 4 y 5 :

Para el cálculo de saturación, se determinó el $R w$ usando un pickett plot de porosidad vs resistividad; obteniéndose para el Ciclo 5 un $R w=0,35$ (Tabla 4).
El Ciclo 4 presenta más intervalos arenosos que el ciclo anterior, para este ciclo se determinó un $R w=0,85$ usando un picket plott (Figura 21). Utilizando las ecuaciones 5 y 6 , se elaboró la Figura 22 que muestra la curva de $S w$ generada para los ciclos 4, 5 y 6 .

Las Tablas 4 y 5 muestran los valores utilizados para el cálculo de saturación de agua por el método de Doble Agua y los valores de $S w$ promedio calculados en las zonas de Net Rock para los ciclos 4, 5, 6y 8, encontrándose un valor promedio de $95 \%$ de saturación de agua.

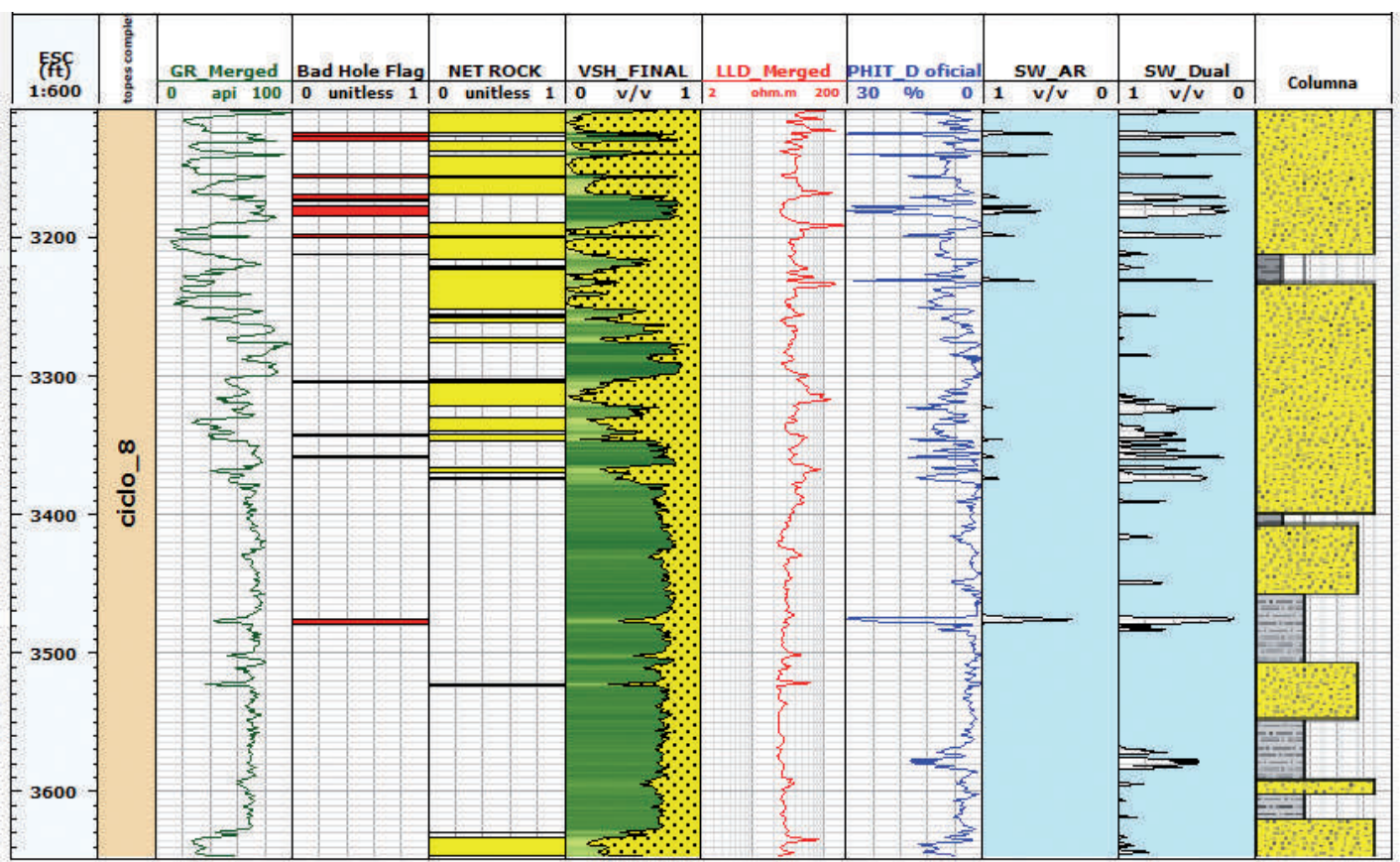

Figura 20. Curva de saturación de agua $(S w)$ y $S w$ Dual (Dual Water) calculada a partir de la porosidad por densidad (PHIT D oficial) y el registro de resistividad profunda (LLD_Merged) para el Ciclo 8.
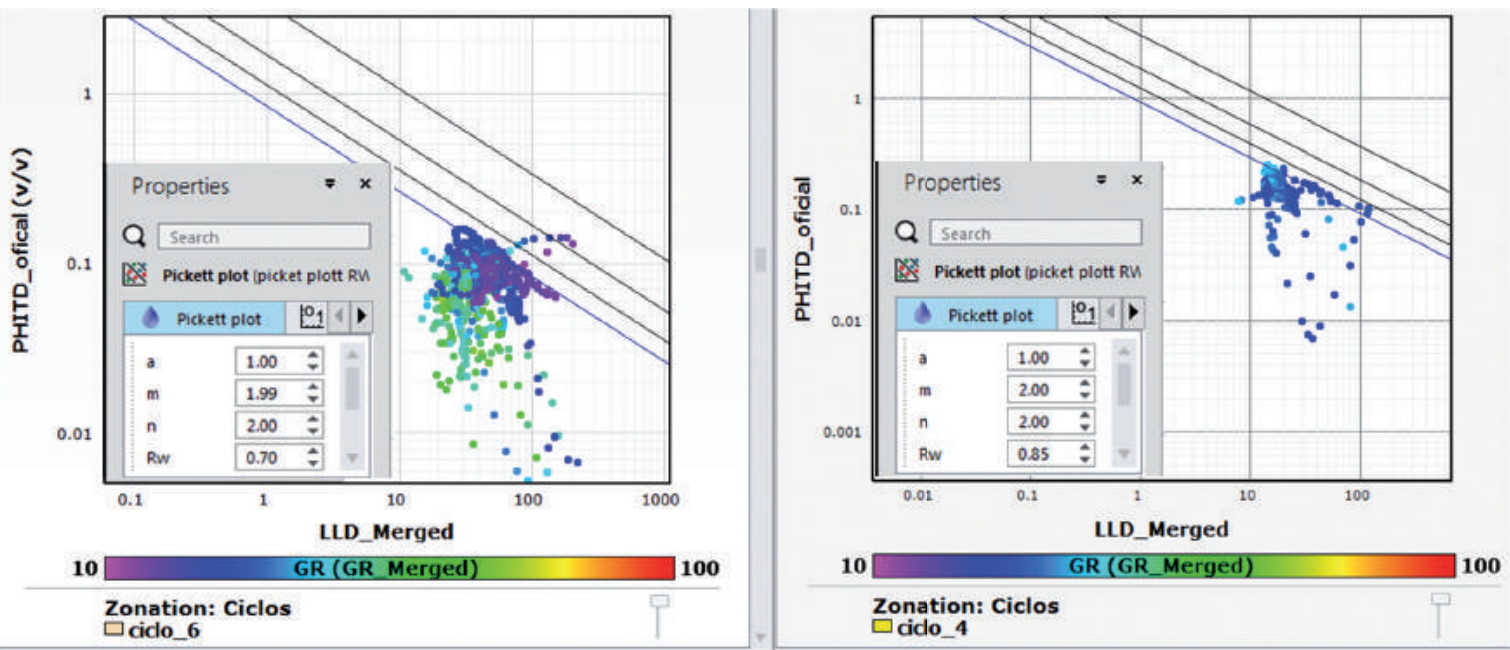

Figura 21. A. Picket plot porosidad (PHIID oficial) vs resistividad (LLD Merged) para el Ciclo 6. B. Picket plot porosidad vs resistividad para el Ciclo 4. 


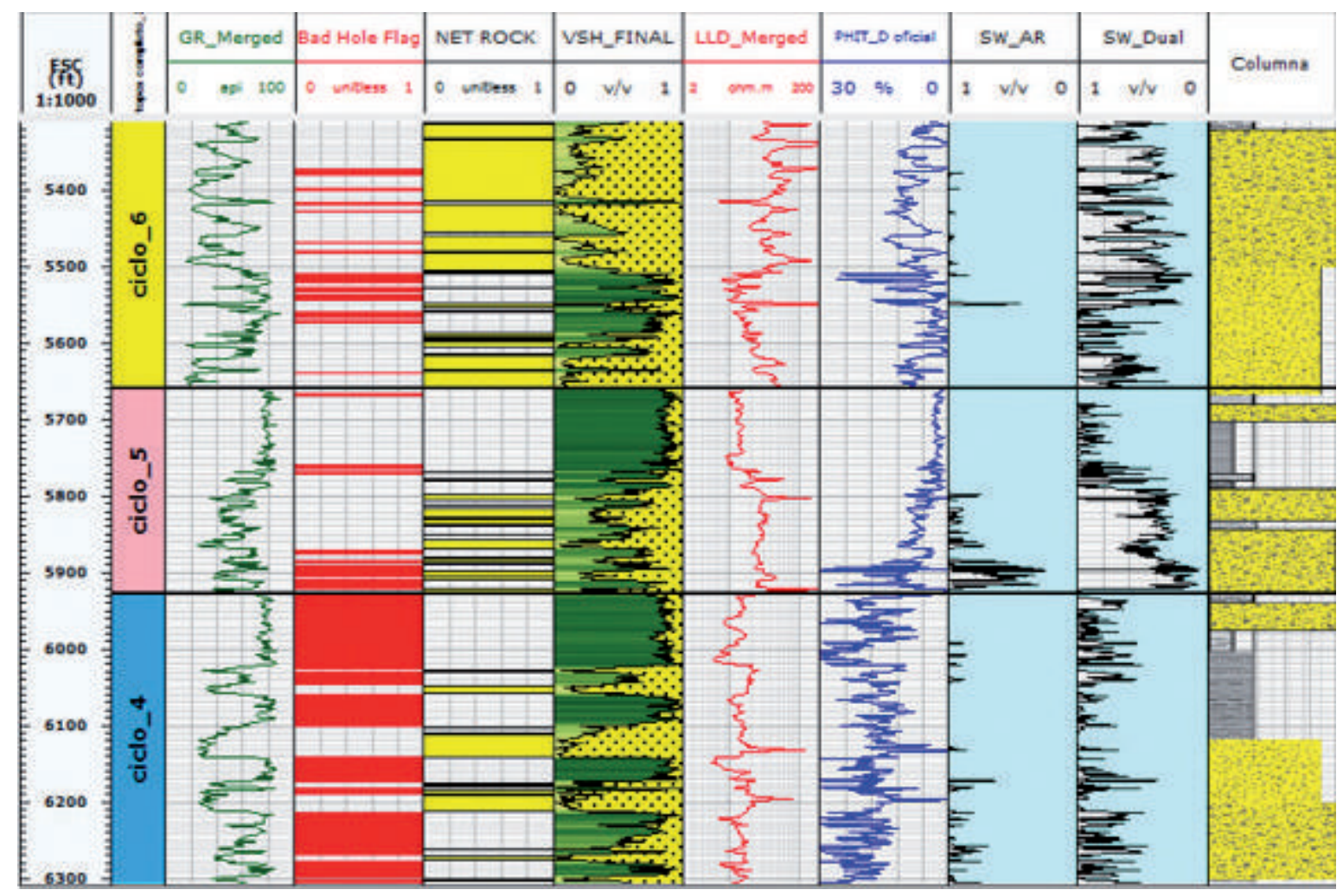

Figura 22. Curva de saturación de agua Sw_AR (Archie) y Sw por Doble Agua (Sw_Dual) para los ciclos 4, 5 y 6.

Tabla 5. Valores de $S w$ promedio calculados en las zonas de Net Rock para los ciclos 4, 5, 6 y 8.

\begin{tabular}{cccc}
\hline Ciclo & Rw (ohm.m) & Sw (\%) & Sw_Dual (\%) \\
\hline 8 & 0,9 & 99,3 & 95,4 \\
6 & 0,7 & 99,4 & 61,1 \\
5 & 0,18 & 87,3 & 43,2 \\
4 & 0,85 & 99,3 & 98 \\
\hline
\end{tabular}

Tabla 6. Resumen de las propiedades calculadas para los ciclos siliciclásticos del pozo ANH-TIERRAKTA-2-X-P.

\begin{tabular}{cccccccccccc}
\hline Ciclo & Tope & Base & $\begin{array}{c}\text { Ref. } \\
\text { unit }\end{array}$ & Gross & Net & $\begin{array}{c}\text { Net to } \\
\text { Gross }\end{array}$ & $\begin{array}{c}\text { Prom } V_{\text {sh }} \\
\text { final }\end{array}$ & $\begin{array}{c}\text { Prom. } \\
\text { PHIT_D } \\
\text { oficial }\end{array}$ & $\begin{array}{c}\text { Prom. } \\
\text { Sw_AR } \\
\text { (\%) }\end{array}$ & $\begin{array}{c}\text { Prom. } \\
\text { Sw_Dual } \\
(\%)\end{array}$ \\
\hline 8 & 3107,16 & 3648,2 & Ft & 541,1 & 157,5 & 0,291 & 0,176 & 0,076 & 99,3 & 95,4 \\
6 & 5292,09 & 5658,2 & Ft & 366,1 & 233,1 & 0,637 & 0,203 & 0,084 & 99,4 & 61,1 \\
5 & 5658,23 & 5927,5 & Ft & 269,2 & 59,51 & 0,221 & 0,319 & 0,085 & 87,3 & 43,2 \\
4 & 5927,52 & 6309,8 & Ft & 382,3 & 85,75 & 0,224 & 0,132 & 0,162 & 99,3 & 98 \\
\hline
\end{tabular}

La Tabla 6 muestra un resumen de las propiedades petrofísicas calculadas para el pozo ANHTIERRALTA-2X-P.

\section{DISCUSIÓN}

Los productos diagenéticos encontrados en el análisis petrográfico para el Ciclo 8 demuestran que en este nivel la secuencia sedimentaria fue sometida a compactación y cementación. La pérdida de porosidad y permeabilidad para este ciclo está afectada principalmente por los efectos de compactación, cementación (alcanzando hasta un $19 \%$ de cemento carbonatado de poros) y poronecrosis durante las etapas de eogénesis y mesogénesis; adicional a estos efectos diagenéticos, la presencia de matriz arcillosa (alcanzando un 55\%) en algunas muestras es otro factor determinante para la pérdida de porosidad y permeabilidad en la roca. La redondez, la esfericidad y la selección de los granos también afectan la porosidad como mencionan Beard 
y Weyl (1973), los granos con alta esfericidad tienden a empaquetarse con un mínimo espacio intersticial, y la porosidad y permeabilidad en muchos casos pueden incrementar o disminuir, dependiendo por supuesto de la fabricación u orientación de los granos. Las secciones delgadas (Figuras 5 y 6) muestran que los granos no presentan ninguna orientación preferencial, es decir, la compactación mecánica no fue tan efectiva en algunas muestras.

La cementación fue dada principalmente en el régimen mesogenético de la diagénesis, la precipitación del cemento carbonatado pudo haber sido influenciada por la interacción de los fluidos intersticiales con la lixiviación de litologías vecinas, en este caso el Ciclo 9 (Figura 3) en lo que es llamado transferencia de masas (Worden y Burley, 2003). Los ciclos siliciclásticos analizados presentan compactación diferencial; este fenómeno ocurre después de la depositación de algunos sedimentos, de modo que las diferentes partes de la acumulación sedimentaria desarrollan diferentes grados de porosidad o se depositan en forma irregular durante el enterramiento por debajo de las capas sucesivas de sedimentos. Esto puede ser el resultado de la localización sobre una superficie irregular, tal como en las proximidades de una estructura de arrecife o sobre ésta, o cerca de una falla de crecimiento, o del diferente grado de susceptibilidad a la compactación (Schlumberger, 2017). Como se ha visto el Ciclo 8 es el más compactado y pudo estar sometido a esfuerzos in-situ anómalos debido a la carga litostática diferencial controlada por la geometría del basamento de la cuenca (Figura 2). El volumen de shale calculado
(Figuras 8, 10, 11, 12 y 14), permitió determinar los niveles más limpios (zonas de Net Rock), en donde se calculó la porosidad y saturación, obteniéndose valores promedio entre 8 y $15 \%$ de porosidad y $95 \%$ de saturación de agua. El Ciclo 8 corresponde a depósitos de planicie mareal (ANH, 2014a), y asociado a esta se presenta shale laminar (Figura 18) siendo este un factor determinante para los altos valores de $V_{s h}$.

Diversos factores tales como contenido de hidrocarburos, zonas de sobrepresión, efectos por el contenido de arcilla (efecto de shale) entre otros (Glover, 2000) alteran las lecturas del registro de densidad, y por ende los valores de la porosidad, lo que explica la presencia de valores bajos de porosidad y la incompatibilidad entre datos de laboratorio y de registro eléctrico.

Las porosidades calculadas en los niveles siliciclásticos basales (Figuras 16 y 17), variando entre 5 y $12 \%$ muestran claramente que estos niveles son los más propicios para la acumulación de hidrocarburos ya que no están compactados ni cementados (ANH, 2014b). Como se mencionó anteriormente, el pozo al estar perforado en el flanco de un pliegue sinclinal, se encuentra en un sistema petrolífero abierto (Figura 1), es decir, no presenta ningún cierre estructural y por lo tanto la acumulación de hidrocarburos es casi nula (Tabla 6). Sin embargo, al observar los pickett-plot de porosidad calculada vs Resistividad profundad en el Ciclo 6 se presenta un nivel en donde la Porosidad es constante (Figura 23) y aumenta la resistividad, esas zonas indican la presencia de hidrocarburo en ese ciclo.

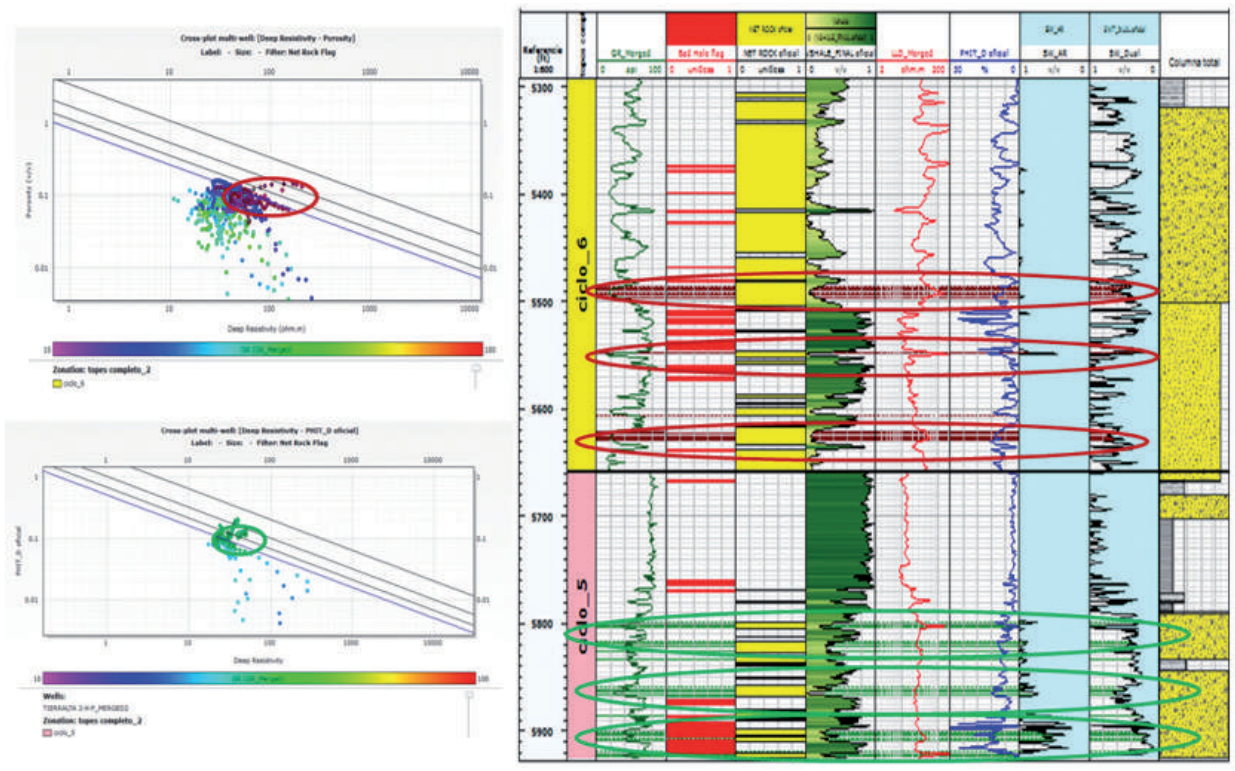

Figura 23. Zonas con acumulación de hidrocarburos en los ciclos 6 y 5 . 
Así mismo el pickett-plot de Porosidad calculada vs Resistividad profundad hecho para el Ciclo 5 muestra el mismo comportamiento en los valores de resistividad vs porosidad, indicando indicios de hidrocarburos.

Del sistema petrolífero de la cuenca, los ciclos basales (ciclos 4,5 y 6 ) son los más favorables para ser rocas reservorio de hidrocarburos, mientras que el Ciclo 7 por sus características litológicas y gran espesor corresponde a un sello en la cuenca; el hidrocarburo puede estar almacenado en trampas estratigráficas donde hay variaciones laterales de facies o en las zonas de los ejes de los pliegues anticlinales.

\section{CONCLUSIONES}

Las características texturales de la roca como el tamaño de grano y la selección, así como los cambios composicionales de la roca, principalmente el contenido de calcita como material cementante y la presencia de shale disperso, tanto estructural como laminar, son factores que afectan significativamente los valores de porosidad y permeabilidad. En particular, el shale laminar es una barrera para el paso de fluidos verticalmente.

El análisis petrográfico hecho para las rocas del Ciclo 8, permitió ubicar las muestras en los campos de cuarzoarenita, litoarenita y sublitoarenita submaduras siguiendo la clasificación de Folk (1974).

Los ciclos siliciclásticos basales (ciclos 4,5 y 6 ) perforados por el pozo ANH-TIERRALTA-2-X-P son los más favorables para ser rocas reservorio de hidrocarburos livianos o gas (hasta un 15\% de porosidad calculada).

El Ciclo 7 es una espesa secuencia de lutitas las cuales representan un sello potencial en el sistema petrolífero.

\section{AGRADECIMIENTOS}

Esta investigación está enmarcada en el proyecto "Estratigrafía del Cretácico superior - Paleógeno de San Jacinto-Caribe colombiano: aporte al conocimiento de su evolución geológica y sistemas petrolíferos" desarrollado por la Agencia Nacional de Hidrocarburos (ANH) y el Instituto de Investigaciones en Estratigrafía (IIES). Agradecemos a la ANH por permitir emplear datos de su propiedad, al IIES por el préstamo de sus instalaciones y equipos y por último al Geólogo Jairo Alonso Osorio y al profesor PhD. Andrés Pardo Trujillo.

\section{REFERENCIAS}

ANH. (2014a). Informe final perforación y geología pozo ANH-TIERRALTA-2-X-P. Agencia Nacional de Hidrocarburos, Bogotá, Colombia.

ANH. (2014b). Pozo: ANH-TIERRALTA-2-X-P. Registro gráfico compuesto. Escala 1:500. Agencia Nacional de Hidrocarburos, Bogotá, Colombia.

ANH. (2016). Nuevas oportunidades exploratorias. Cuenca Sinú-San Jacinto. Agencia Nacional de Hidrocarburos, Bogotá, Colombia.

Archie, G.E. (1941). The electrical resistivity log as an Aid determining some reservoir characteristics. Transactions of the AIME, 146(1), 54-62. doi: 10.2118/942054-G.

Alfaro, E., and Holz, M. (2014). Review of the chronostratigraphic charts in the Sinú-San Jacinto basin based on new seismic stratigraphic interpretations. Journal of South American Earth Sciences, 56, 139-169. doi: 10.1016/j. jsames.2014.09.004.

Beard, D.C., and Weyl, P.K. (1973). Influence of texture on porosity and permeability of unconsolidated sand. AAPG Bulletin, 57(2), 349-369.

Beroiz, C., Linderberg, F., and Winter, S.R. (1986). Northwest Colombia Hydrocarbon Evaluation. Informe Chevron Overseas Petroleum, Inc.

Bermúdez, H.D., Alvarán, M., Grajales, J.A., Restrepo, L.C., Rosero, J.S., Guzmán, C., Ruíz, E.C., Navarrete, R.E., Jaramillo, C. y Osorno, J.F. (2009). Estratigrafía y evolución geológica de la secuencia sedimentaria del Cinturón Plegado de San Jacinto. XII Congreso Colombiano de Geología, Paipa, Colombia.

Burley, S.D. (1984). Patterns of diagenesis in the Sherwood Sandstone Group (Triassic), United Kingdom. Clay Minerals, 19(3), 403-440. doi: 10.1180/claymin.1984.019.3.11.

Buryakovsky, L., Chilingar, G.V., Rieke, H.H., and Shin, S. (2012). Fundamentals of the petrophysics of oil and gas reservoirs. New Jersey: John Wiley \& Sons. 
Cobb, W.M., and Marek, F.J. (1998). Net pay determination for primary and waterflood depletion mechanisms. SPE Annual Technical Conference and Exhibition. New Orleans, USA.

Duque-Caro, H. (2000). Análisis bioestratigráficos de 400 muestras de 34 pozos y 16 muestras de superficie de las cuencas San Jorge, Sinú, Plato y Barranquilla, en el Valle Inferior del Magdalena. Reporte interno, ECOPETROL, Colombia.

Folk, R.L. (1974). Petrology of sedimentary rocks. Austin: Hemphill Publishing Company.

Glover, P.W.J. (2000). Petrophysic. Msc Course Notes. Aberdeen: University of Aberdeen.

Guzmán, G. (2007). Stratigraphy and sedimentary environment and implications in Plato basin and the San Jacinto Belt Northwestern Colombia. Ph.D. Thesis, University of Liége, Belgium.

Guzmán, G., Gómez, E., y Serrano, B. (2004). Geología de los cinturones del Sinú, San Jacinto y borde occidental del valle inferior del MagdalenaCaribe Colombiano. Bogotá: INGEOMINAS.

Kennedy, M. (2015). Practical Petrophysics. Vol. 62. Elsevier.

Pickett, G.R. (1973). Pattern recognition as a means of formation evaluation. The Log Analyst, 14(04), 3-11.

Schlumberger. (1990). Principios/análisis de la interpretación de registros. Houston: Schlumberger.

Schlumberger. (1996). Introduction to open hole logging. Houston: Schulmberger.

Schlumberger. (2013). Techlog Fundamentals Workflow/Solutions Training. Houston: Schulmberger.
Schlumberger. (2017). Compactación diferencial. Retrieved from: http://www.glossary.oilfield.slb. com/es/Terms/d/differential_compaction.aspx

Spooner, P. (2014). Lifting the fog of confusion surrounding clay and shale in petrophysics. SPWLA 55th Annual Logging Symposium. Abu Dhabi, United Arab Emirates.

Universidad de Caldas-ANH. (2011). Estudio integrado de los núcleos y registros obtenidos de los pozos someros (slim holes) perforados por la ANH. Contrato 093 de 2009. Manizales, Colombia.

Villafrade, Y.G. (2007). Estratigrafía y sedimentación en el Eoceno, para los pozos PORVENIR-3 y Cerro Villanueva 1, en el área del Cinturón de San Jacinto, Valle Inferior del Magdalena, NW de Colombia. Tesis, Universidad Industrial de Santander, Bucaramanga, Colombia.

Viro, E. (1997). Interpretación de registros a agujero descubierto. Río de Janeiro: Viro Consultores Ltda.

Worden, R.H., and Burley, S.D. (2003). Sandstone diagenesis: the evolution of sand to stone. In: S. Burley, R. Wonden (eds.). Sandstone diagenesis: recent and ancient (pp.1-44). International Association of Sedimentologists. doi: 10.1002/9781444304459.ch.

Cristian Camilo Manrique Gómez

ORCID: 0000-0002-0147-0683

Carlos Alberto Guzmán López

ORCID: 0000-0001-6658-8909

Robinson Aguirre

ORCID: 0000-0001-5246-3187

Trabajo recibido: agosto 29 de 2018

Trabajo aceptado: noviembre 28 de 2019 